\title{
SOME UNPUBLISHED RELIGIOUS TEXTS OF ŠMAM̆
}

\author{
By Clifton Daggett Gray, \\ The University of Chicago.
}

Until within a comparatively short time the publication of Assyrian Religious Texts has been of a desultory character, few attempts having been made systematically to bring out complete series of texts. Among continental scholars three marked exceptions may be mentioned. To Tallqvist for his publication of Die assyrische Beschwörungsserie Maqla, and to Zimmern for his publication of Beiträge zur Kenntnis der babylonischen Religion, as well as to Knudtzon for his Assyrische Gebete an den Sonnengott, students of Assyrian and Babylonian religion are deeply indebted. Among English Assyriologists a similar debt is due to King for his careful editing of The Prayers of the Lifting of the Hand, and to Thompson for The Reports of the Magicians and Astrologers of Nineveh and Babylon. Such work as these men have done has been greatly facilitated by the appearance, two years ago, of the fifth volume of the Catalogue, but there remains a vast amount of material yet to be brought to light. In his preface to Babylonian Religion and Mythology, King well says: "Although so much has been done in recent years to explain their religious literature, no finality in the matter must be expected for some time to come, certainly not as long as any important religious text remains unpublished." For this reason, then, these religious texts relating to the worship of Šamaš have been published, in the hope that they may form a small contribution to the history of Babylonian religion, the writing of which, as Zimmern has remarked in the Vorwort of his Beiträge zur Kenntnis der babylonischen Religion, is "today as yet a thing impossible."

The texts which are given in the following pages comprise all of the hitherto unpublished Samaš Religious Texts, which are classified in the fifth volume of the Catalogue of the Cuneiform Tablets in the Kouyunjik Collection of the British Museum as "Incantations and prayers and hymns to the Sun-god" (p. 2053), 
as "Hymns to Šamaš" (p. 2044), and as "Prayers to Samaš" (p. 2153). For two reasons it was not thought best to include those texts which have already been published. In the first place, with two or three exceptions they are all given in the second edition of Vol. IV of the Cuneiform Inscriptions of Western Asia, which means that very few corrections to these plates could be found. Secondly, the present piece of work is only preliminary to a more extended discussion of the Samaš Religious Literature, which will include a transliteration and translation of all the Samaš texts, together with exegetical notes and glossary, and an introduction. In the case of one text already published, K. 3182, so many fragments have been added to it as to make it almost a new text. For this reason, and also the fact that it is one copy of a very important hymn, it has been included. In the case of K. 4872 , the numerous duplicates of this valuable incantation text have been published, thus making possible the restoration of a large portion of the tablet, although the incantation to Samaš occupies only the first column and half of the second.

The greater number of the tablets are in the Assyrian character, and are quite easily read where not mutilated. In one or two tablets, as for example in K. 3286, some of the characters approach the Babylonian. Seven tablets, K. 2132, K. 2296, K. 2883 , K. 4795 , K. 5982, K. 10527, and Bu. 91-5-9, 132, are written in the Babylonian.

As for the language, it is for the most part Assyrian. The following texts are bi-lingual: K. $2605, \mathrm{~K} .4872$ and duplicates, K. 5982 and duplicate, K. 11789 duplicate of K. 4803, S. 690, Rm. 129 duplicate of $\mathrm{K}$. 3343, Bu. 91-5-9, 180 duplicate of K. 256. Two of the texts, K. 3462 and K. 4795, are Sumerian. With regard to the classification given in the Catalogue, it must frequently be considered as merely tentative. For instance, K. 11768 is classified as a hymn, and K. 9830 as an incantation and prayer, but they have both been joined. In many cases, owing to the smallness of the fragment or to the indefiniteness of the contents, it was impossible to give it a more definite classification than that of a "religious text." Again, in the case of many tablets, the classification into incantations, prayers, and hymns is a purely arbitrary one, the dividing line between such religious texts being very vague. A single text will often partake of the character of both a hymn or prayer and an incantation, or 
even of all three of these. There is only one pure hymn among the Samaš Religious Texts.

Three tablets, K. 5900, K. 12000, and Rm. 601, are quite different in the phraseology of the first line from any other Samaš religious text. They all begin: ilu Šamaš bêl di-nim. Comparing this with the first line of the prayers to Rammân (Adad) and Samaš, it is probable that these tablets should be classified under the latter head. In the first line of $\mathrm{Rm}$. 601 enough of the name of the god Rammân (Adad) appears to make this quite certain as far as this tablet is concerned. In a few tablets, $\mathrm{K}$. 3204 , K. 3214, K. 3928, K. 3286, and K. 3394, so much of ritual appears that it would seem better to put them under some other classification, such as "Prayers and Directions for Ceremonies."

The following joins were made: (1) K. $4654+\mathrm{Rm} .2$, 213 ; (2) K. 4922 +K. 11953 ; (3) K. 9830 + K. 11768 ; (4) S. 690 +S. 2070. From a careful comparison of the following duplicates of K. 4872 it is probable that they are fragments of the same tablet, K. $4922+$ K. 11953 and K. 5069 being on the obverse, and K. 5248 and K. 8934 on the reverse. For the same reasons K. 3214 and K. 3928 are evidently portions of the same tablet. K. 13256 is possibly a fragment of the same tablet as K. 8457. K. 10527 is a duplicate of K. 5982, a fact which is not noted in the Catalogue.

In a number of places which are marked in the text as scratched it was almost impossible to ascertain the true reading, owing to the careless cleaning of the tablets by former readers, a practice which was by no means confined to this class of literature, as will be seen by reading the closing paragraph of Thompson's review of Harper's Assyrian and Babylonian Letters, in AJSL., April, 1901, p. 167.

The Samaš Religious Texts which have already been published are as follows: K. 256 in IV R., 2 d ed., plate $17 ; \mathrm{K} .2860$ in IV R., $2 \mathrm{~d}$ ed., plate 19, No. 2 ; K. 3343 in IV R., $2 \mathrm{~d}$ ed., plate 20, No. 2 ; K. 4803 in IV R., 2 d ed., plate 28 , No. 1 ; K. 4872 in V R., plates 50 and $51 ; 33328$ in Abel and Winckler's Keilschrifttexte, pp. 59, 60.

For convenience, a list of duplicates in the following texts is added: of K. 256: Bu. 91-5-9, 180; of K. 3182 : S. 1033, $83-1-18,472$; of K. 3343 : Rm. 129 ; of K. 4803 : K. 11789; of K. 4872 : K. 3138 , K. 3462 , K. 4610, K. 4654+Rm. 2, 213, 
K. 4830 , K. $4922+$ K. 11953 , K. 4986, K. 5069, K. 5135, K. 5248 , K. 8934 , S. 166 , S. 728 ; of K. 5982 : K. 10527 ; of S. $787:$ K. $8457+$ K. 8926.

I wish to thank Dr. Weissbach, of Leipzig, for his kindness in pointing out to me three duplicates of K. 4872 , viz., K. 3138 , K. 3462 , and K. 4654, and another Samaš text, K. 2380, none of which are noted in the Catalogue.

I am also under obligations to Dr. E. Wallis Budge, the Keeper of the Department of Egyptian and Assyrian Antiquities, British Museum, and to Mr. Leonard W. King and Mr. R. Campbell Thompson, assistants in the Department, for their courtesy and for the great assistance which they afforded me during my stay in London.

I am especially indebted to my instructor, Professor Robert Francis Harper, for valuable suggestions and help in my study of these texts. For the results as presented, however, I alone am responsible.

INDEX.

\begin{tabular}{|c|c|c|c|c|c|c|c|c|c|}
\hline & & & & Plates & & & & & \\
\hline K. 273 & & - & & XII & K. 5248 & & & & \\
\hline R. 2132 & - & - & - & XII & K. 5900 & & - & & \\
\hline 96 & & - & 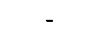 & XIX & K. 5 & & & & \\
\hline 2380 & & & - & III & K. 6 & & & & \\
\hline 2563 & & - & 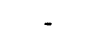 & V & K. 8457 & & & & \\
\hline 2565 & - & - & - & IX & K. 8 & & - & & \\
\hline 2 & & - & 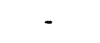 & IX & K. & & & & \\
\hline 83 & & - & - & XII & K. 9 & & - & & \\
\hline 38 & & & & $\mathrm{XVI}$ & K. 1 & & & & \\
\hline & & - & - & I, II & $39-$ & & - & & \\
\hline & & & & VIII & K. & & & & \\
\hline 4 & - & - & - & VII & 256 - & - & - & & \\
\hline & & - & - & III & S. 1 & 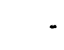 & & & \\
\hline & & & 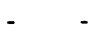 & $\mathrm{X}$ & S. 690 & & & & \\
\hline & & - & & VII & S. & & & & \\
\hline$?$ & & & - & $\mathrm{XV}$ & S. 7 & & & & \\
\hline & & - & - & VII & & 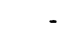 & & & \\
\hline 4 & - & - & - & XVIII & S. 1310 & & - & & \\
\hline & & & - & - $\quad X V$ & S. 1 & & & & \\
\hline & & - & & XIII & & - & - & & \\
\hline & & & - & XIV & 301 & & & - & \\
\hline & 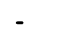 & - & & XIV & 8,472 & & - & & \\
\hline & & & & XVII & $5-9,132$ & & & & \\
\hline & 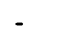 & - & & XV & Bu. 91-5-9, 180 & & - & & \\
\hline 5 & & & - & XVII & & & & & \\
\hline
\end{tabular}




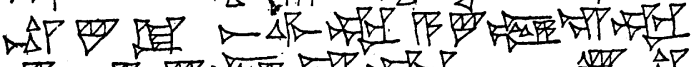
运

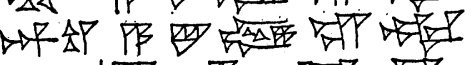

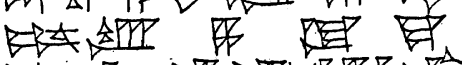
A

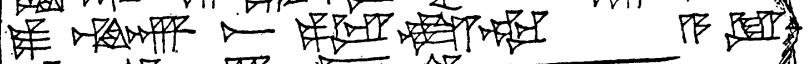

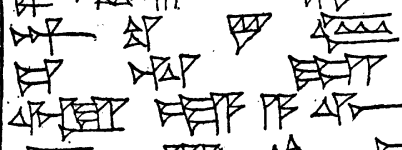

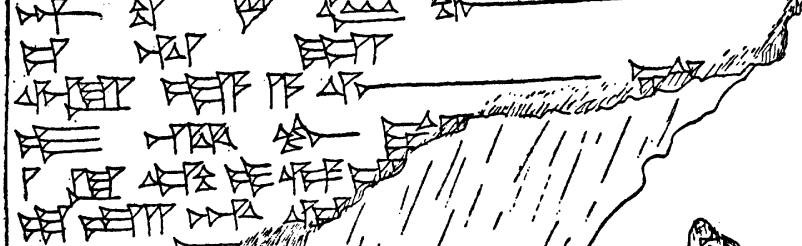

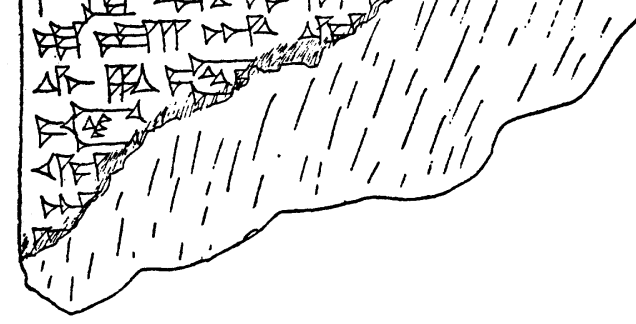

K3286. Obr.
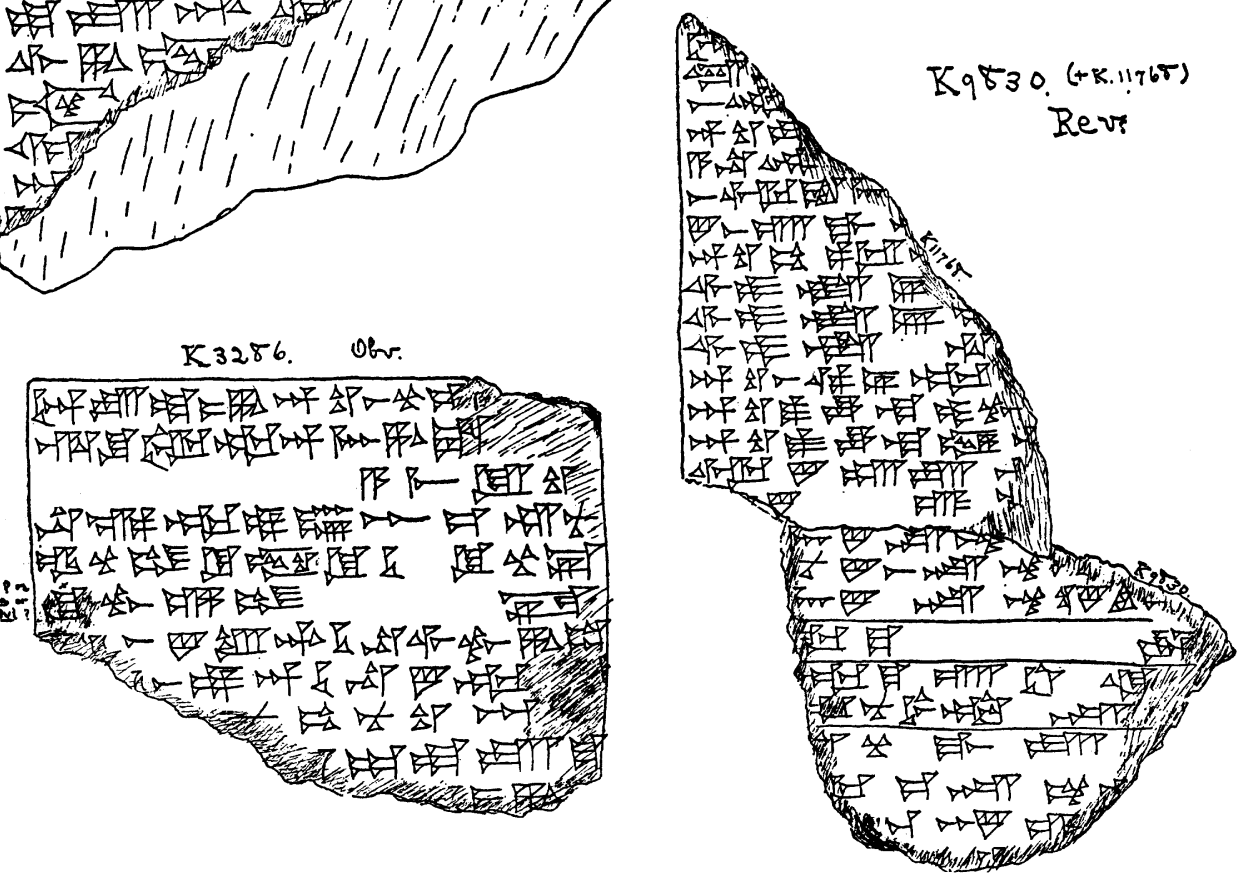

[HEBRAICA, XVII] 
Plate II.

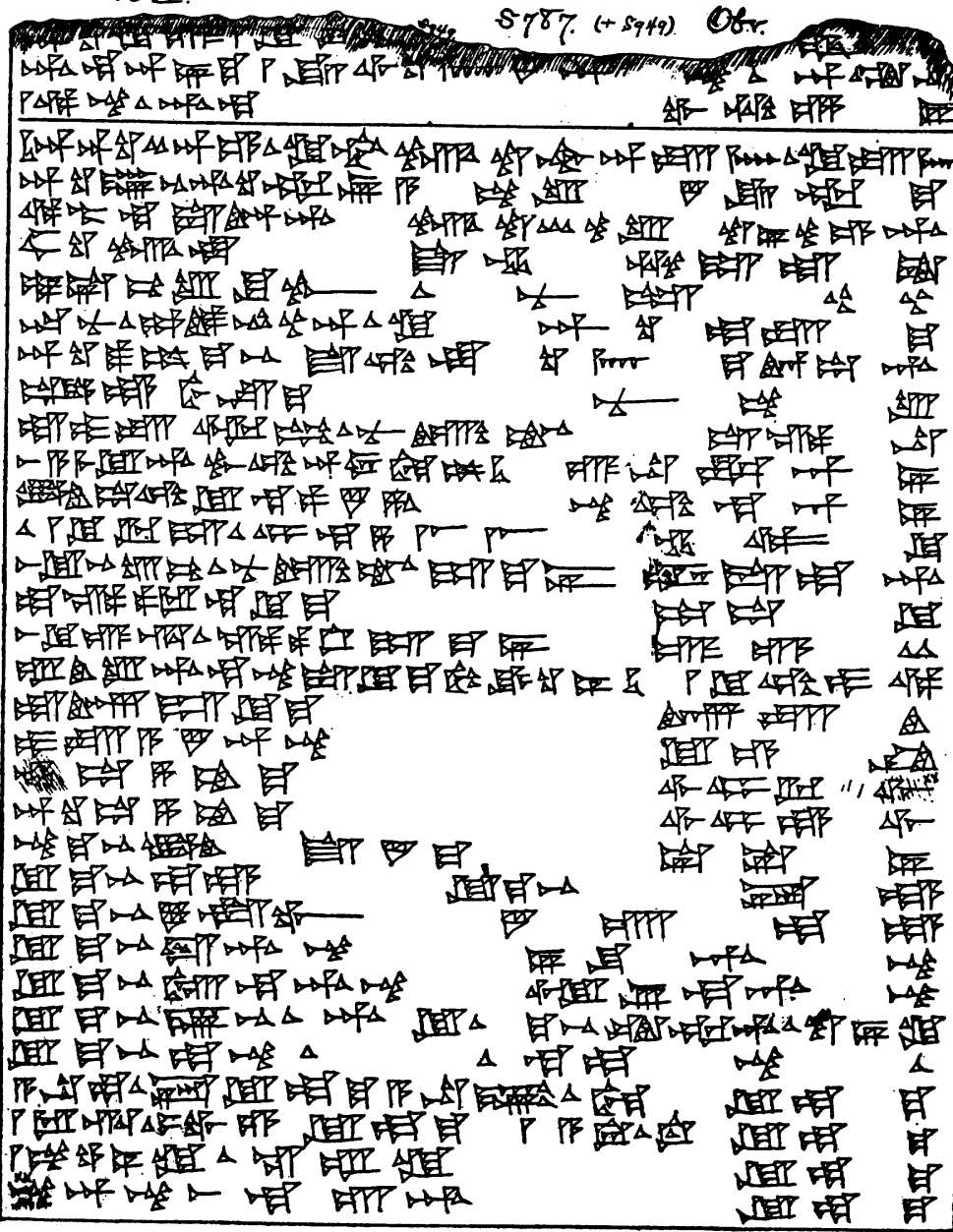

xxceratehed

$$
\text { Rev. }
$$

sma

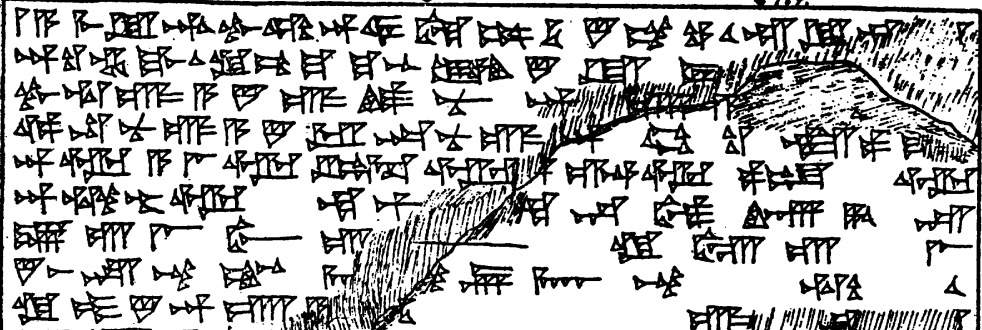

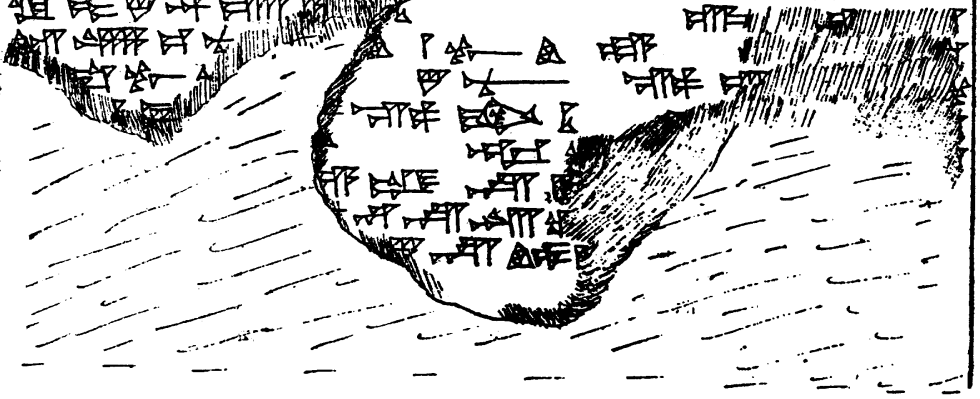


Plate

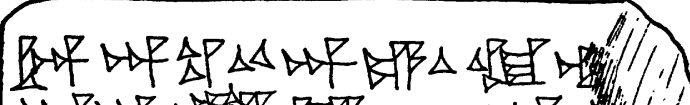
UF

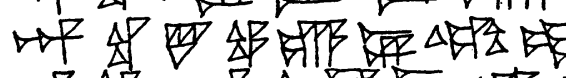

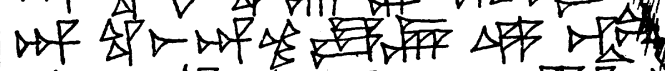

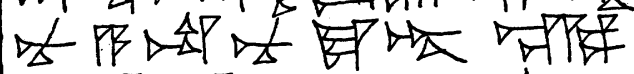

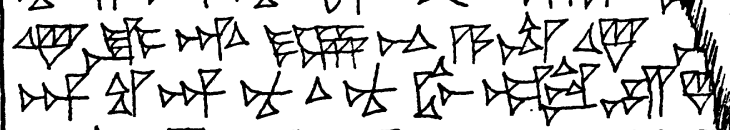

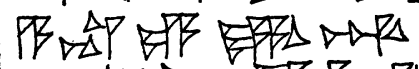

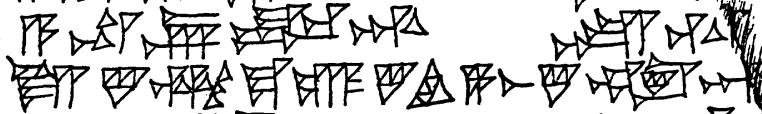

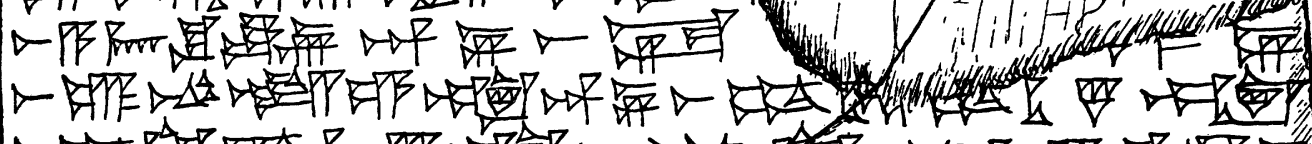

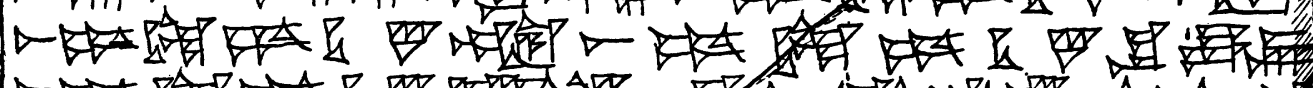

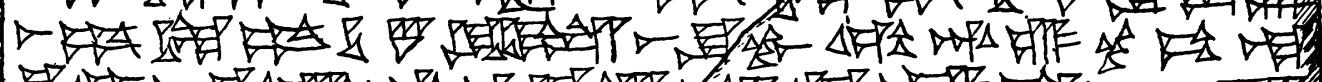

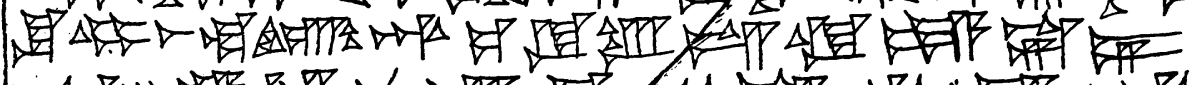

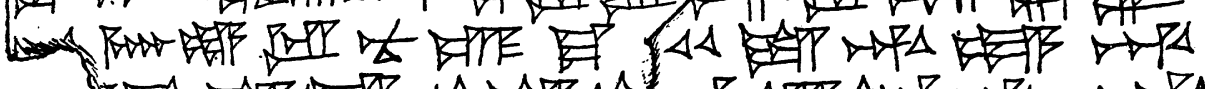

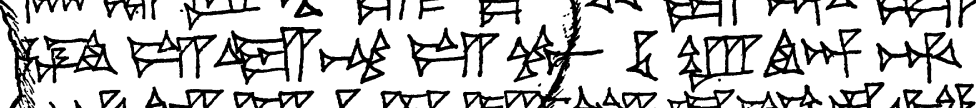

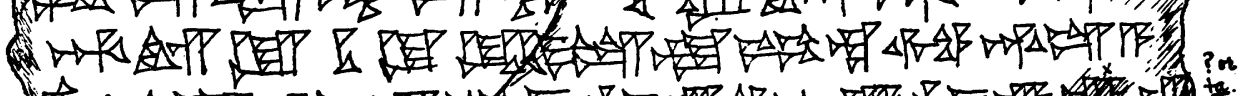

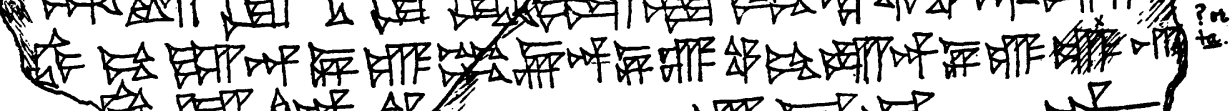

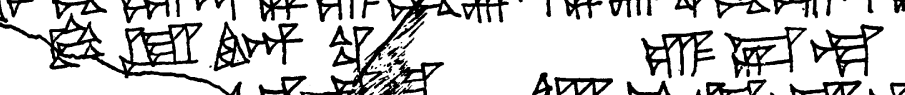
U, 邻 $\rightarrow 7$

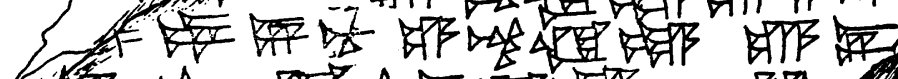
1 管

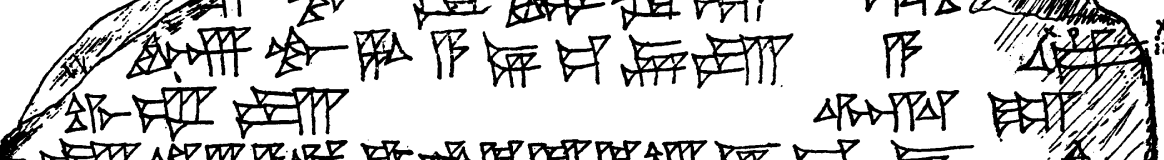
望

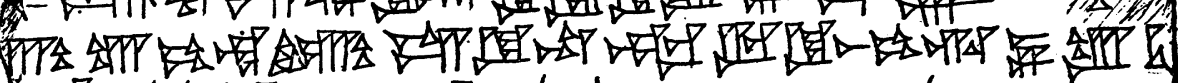
$\Delta \sim 7 \Delta \Delta, \Delta \Delta \forall \Delta$

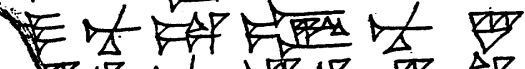

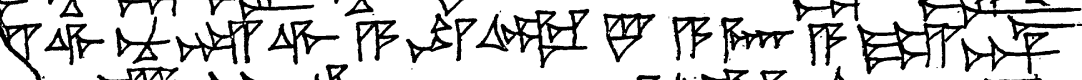

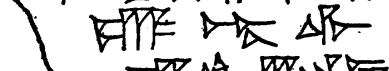

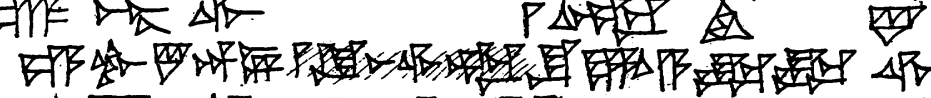
$=0 T$ TSA

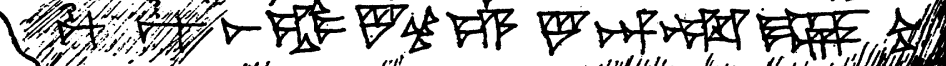

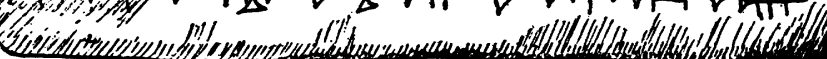



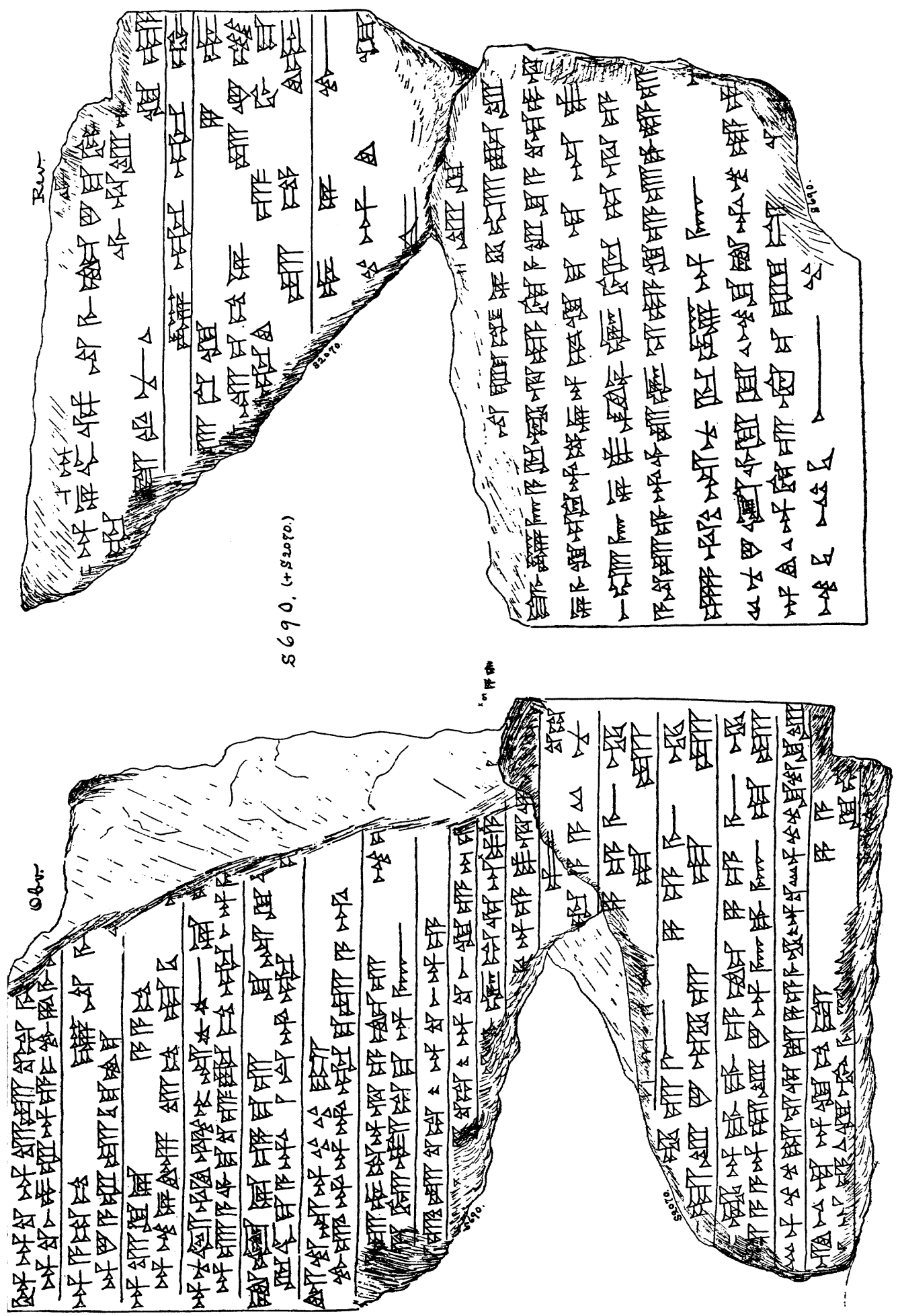


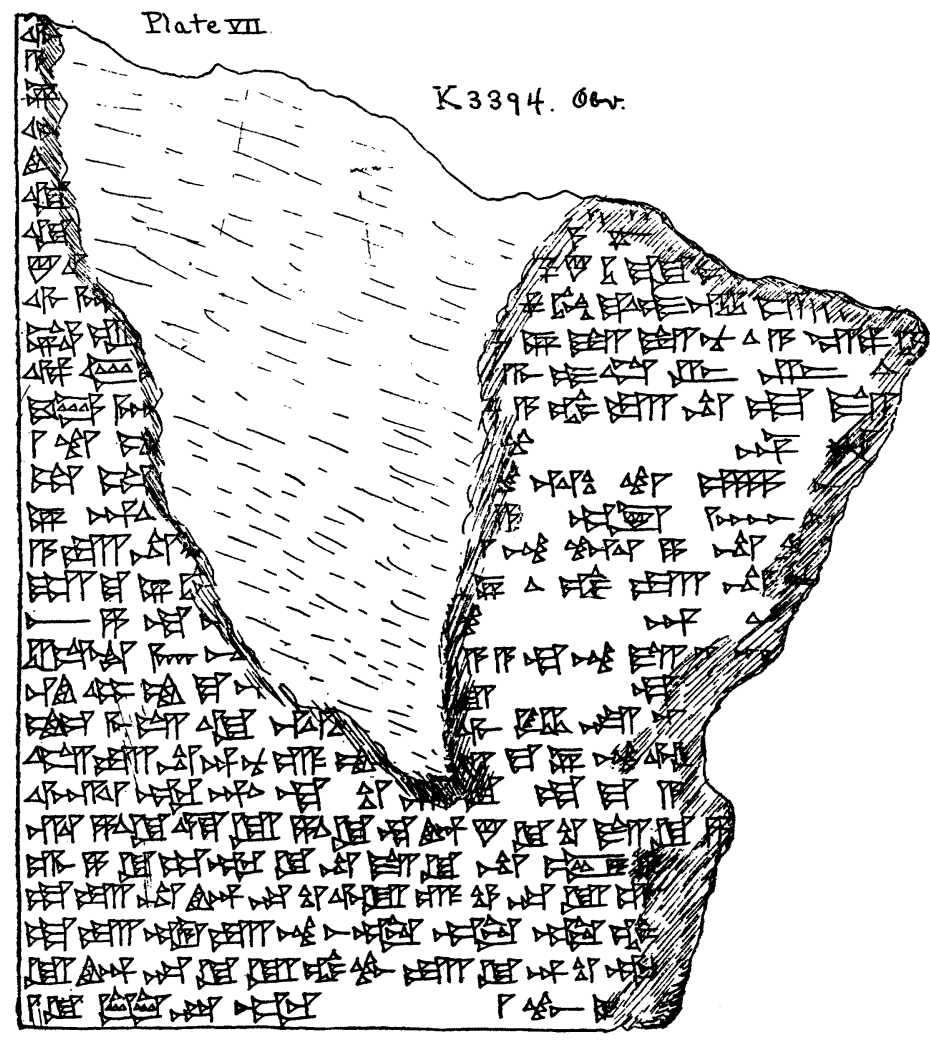

K3394. Rev
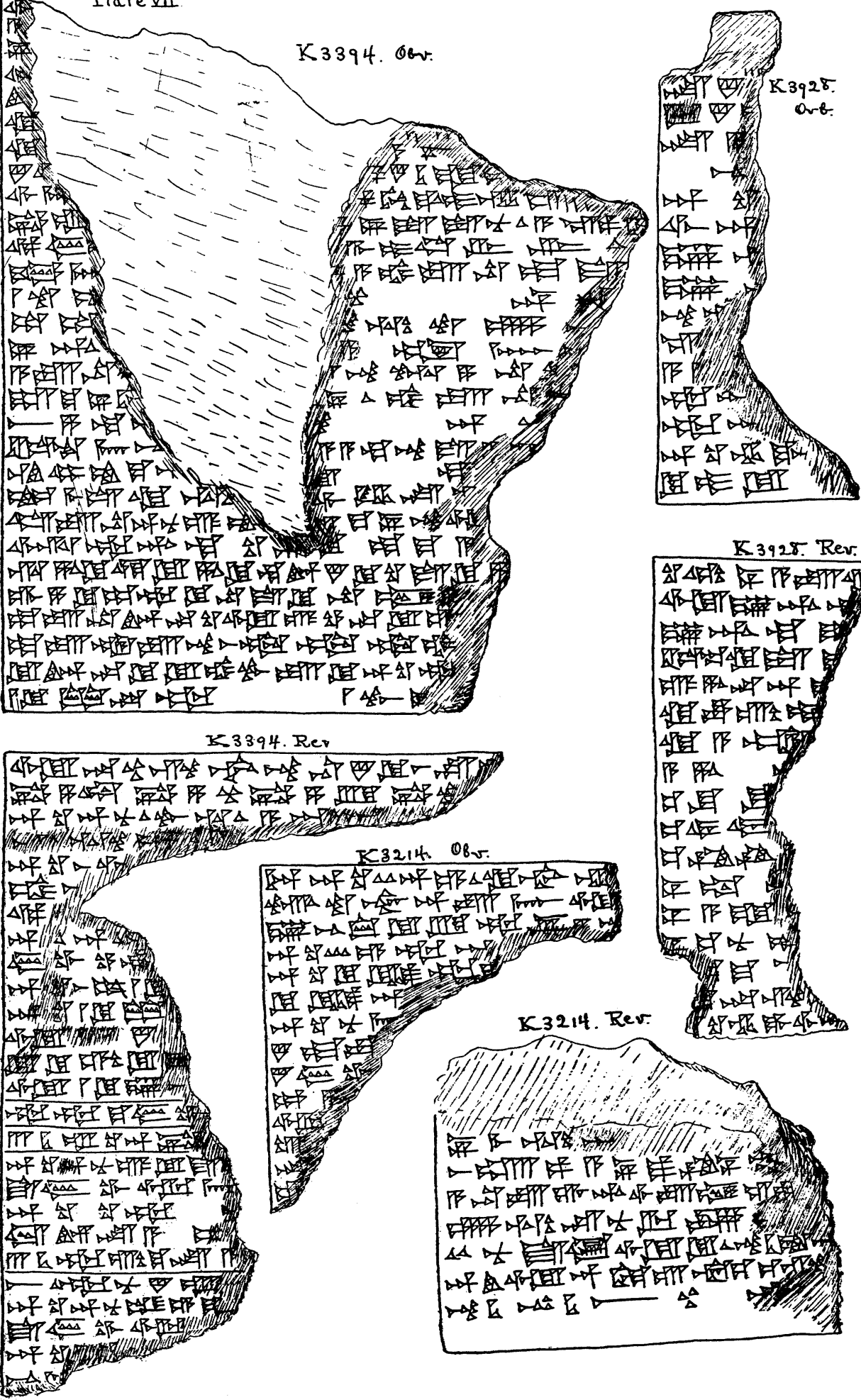

K.3928. Rev.

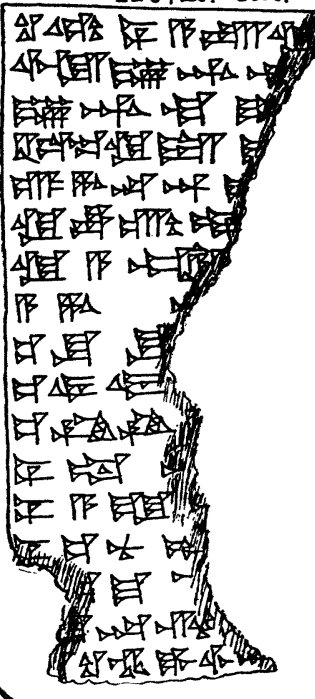

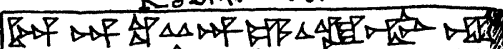
SATR

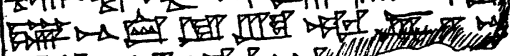

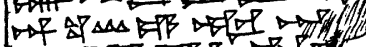

$\rightarrow \rightarrow$ 邻最

促

$\rightarrow 7$ 난

K.3214. Rer.

(1)

$7 \rightarrow$

17

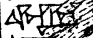

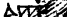

$\rightarrow$

卒

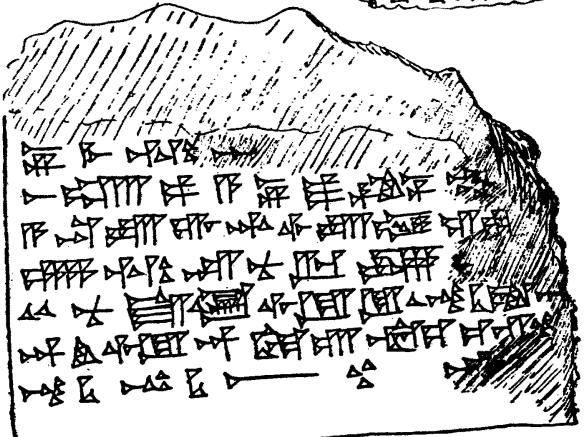



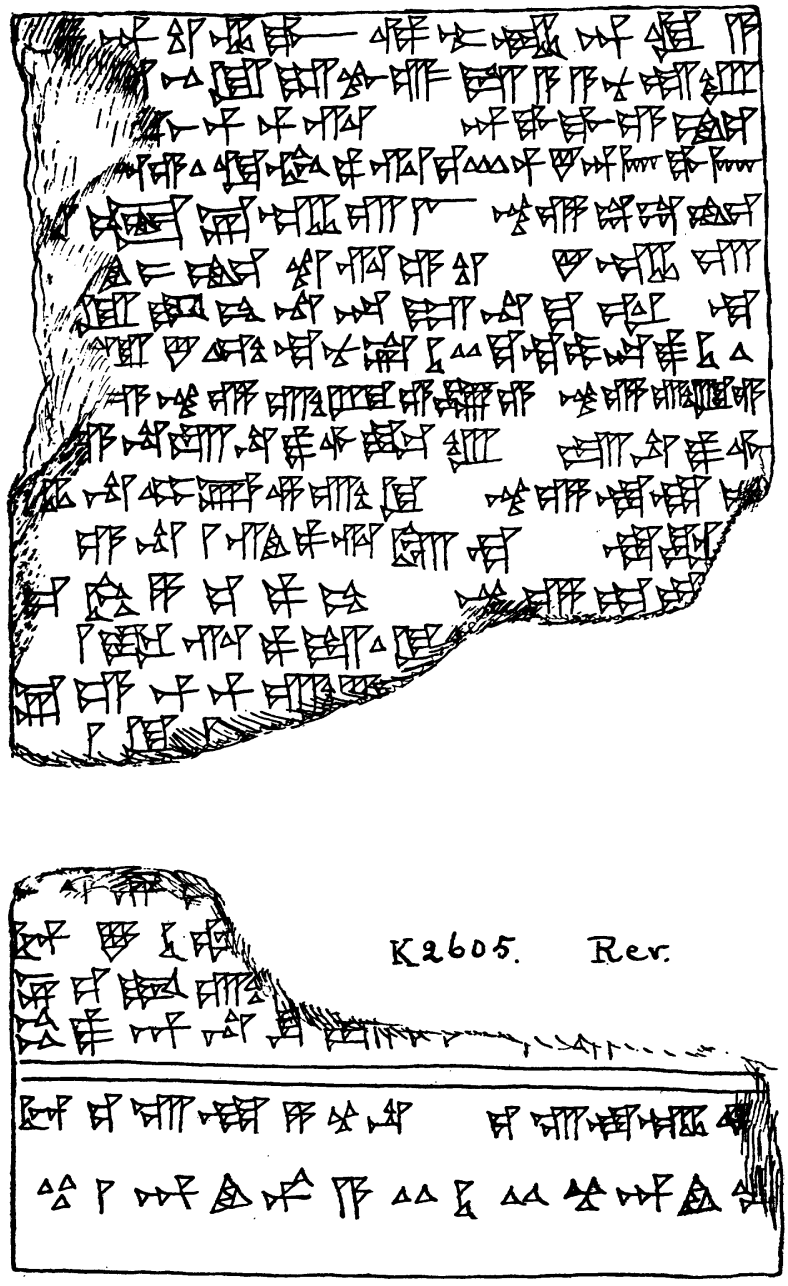

K2565.

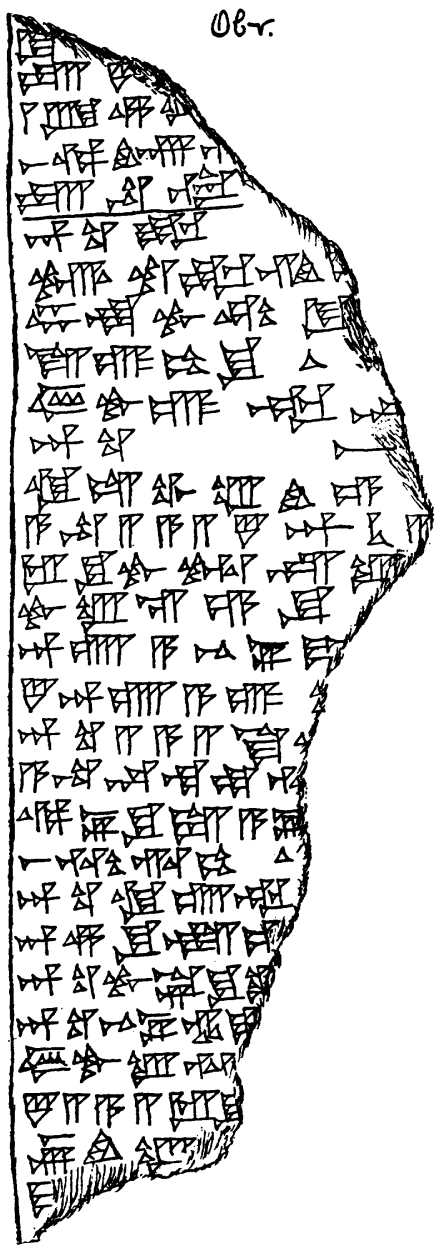

$\mathrm{K} 6034$. obr. 61 140 作

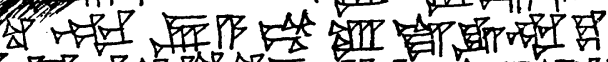

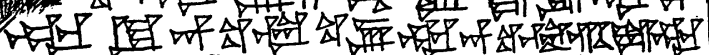
1 OP $\Delta P$ T

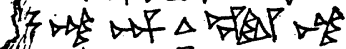

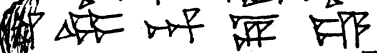
布隔 留 4h

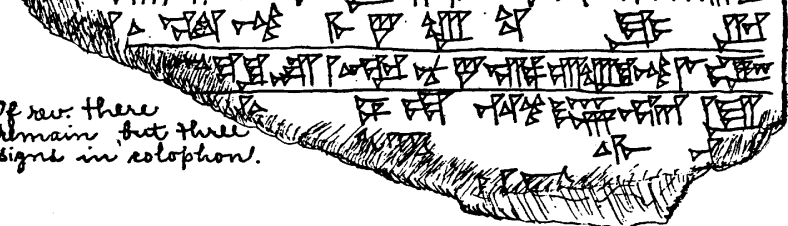

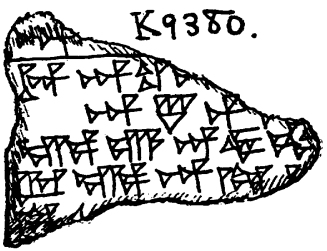


Plate $X$

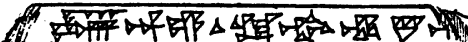

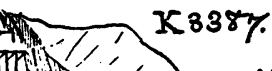

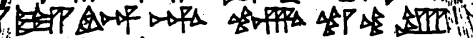

Pas

MU

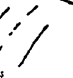

min

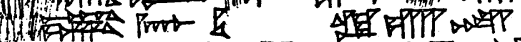

His 11

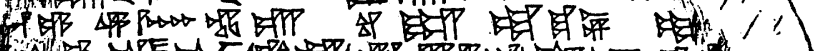

14 A H

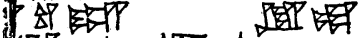

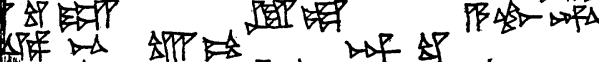

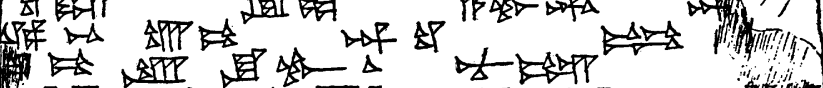

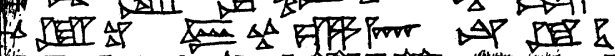

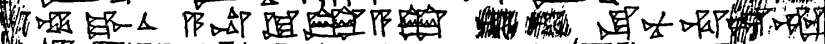
\%

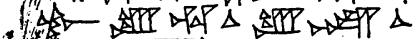

12

湾 $\Delta \nabla$

in)

\section{hitis}

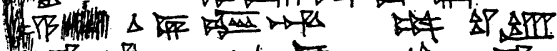
4.

Wif

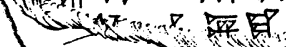

S

M

保 还

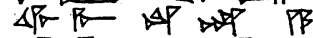

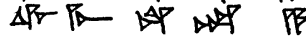

年

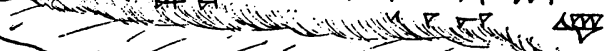

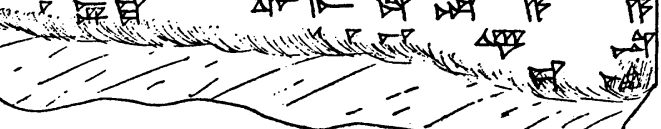

Bear.

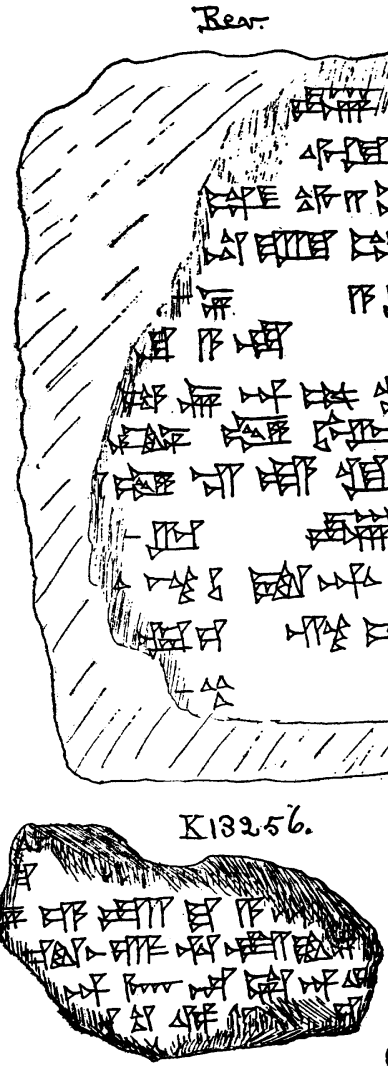

Tit

$\widehat{\sigma e}$

पस

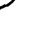

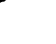

计为宜 M. 4 Ff

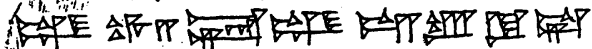

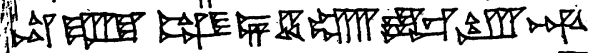

6)

P 不

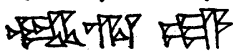

䏝

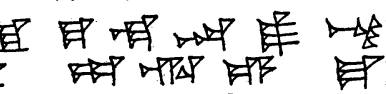

ATP ATP

काT

DP

(II) -

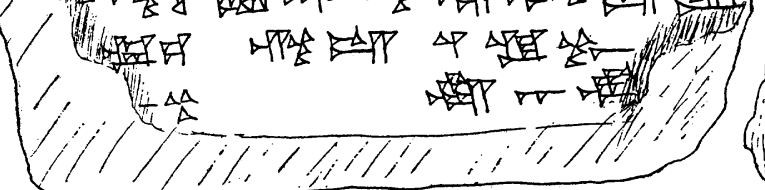

[Hebraica, XVII]

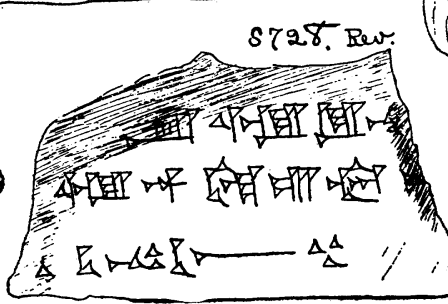

s728. Rew

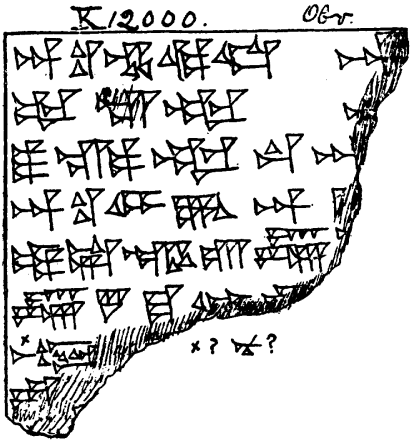

Rev

समाT

*

$\triangle$ 原

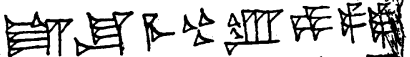

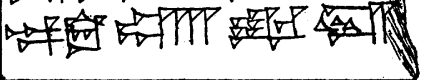

$5 / 310$.

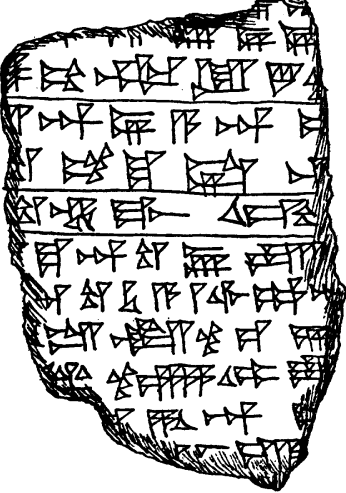

572.8 .060$.

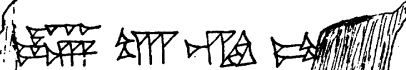

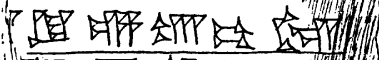

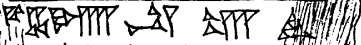

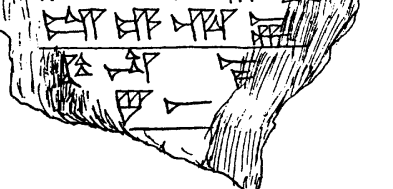

This content downloaded from 129.219.247.033 on September 01, 2016 14:07:24 PM 


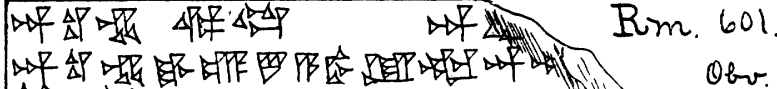

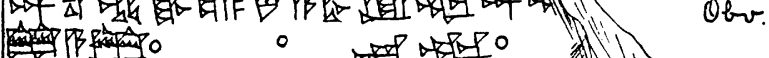

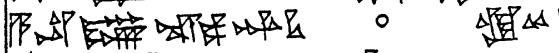

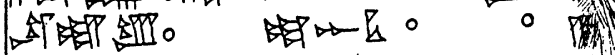

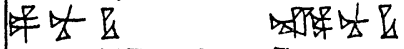
ARP $\Delta$ NPP $\rightarrow P$ \& 故公

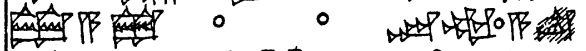
BSP

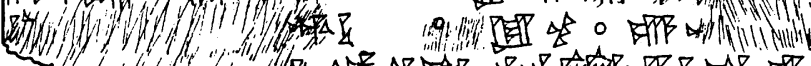
VIIII

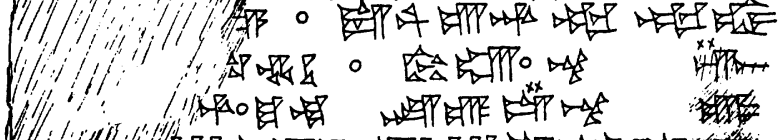
(y)

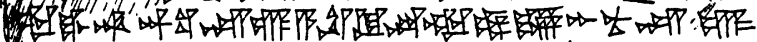

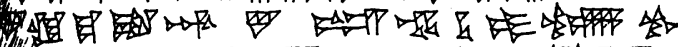

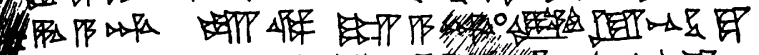

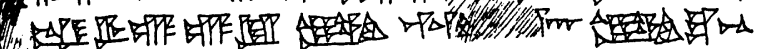

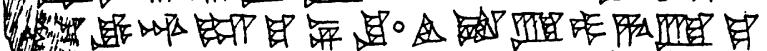
4itis

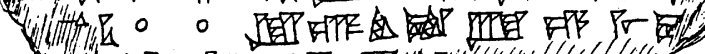
* ${ }_{\text {scrutched }}$

$$
\text { sx scratched }
$$

Rev Th

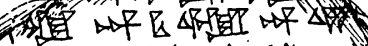

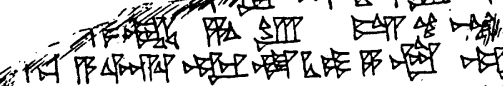

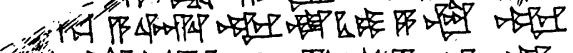

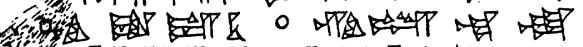

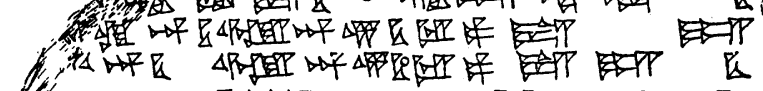
BPAD OF

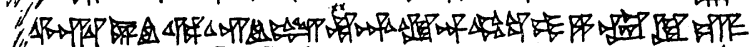
13\%? 还 -

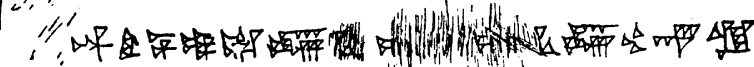

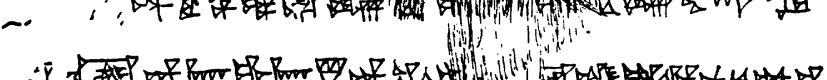

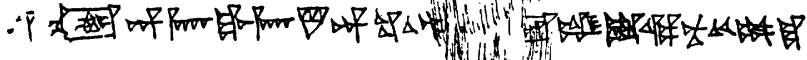

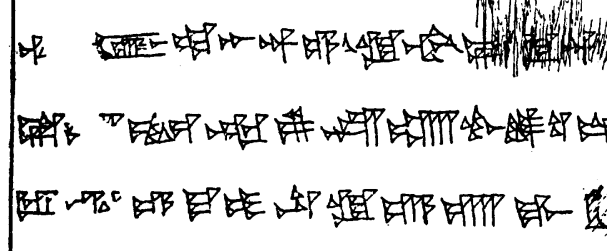


Plate XII.

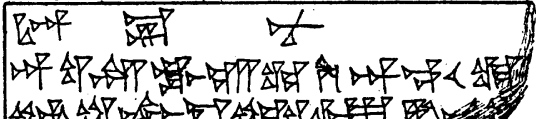

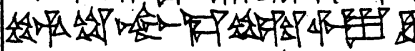

सR

A

- 跑

우 $\frac{\Delta}{4} \nabla$ 邻展

为作邻

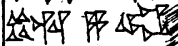

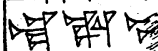

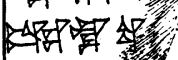

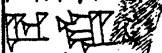

77040

$\forall$

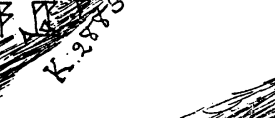

4. -
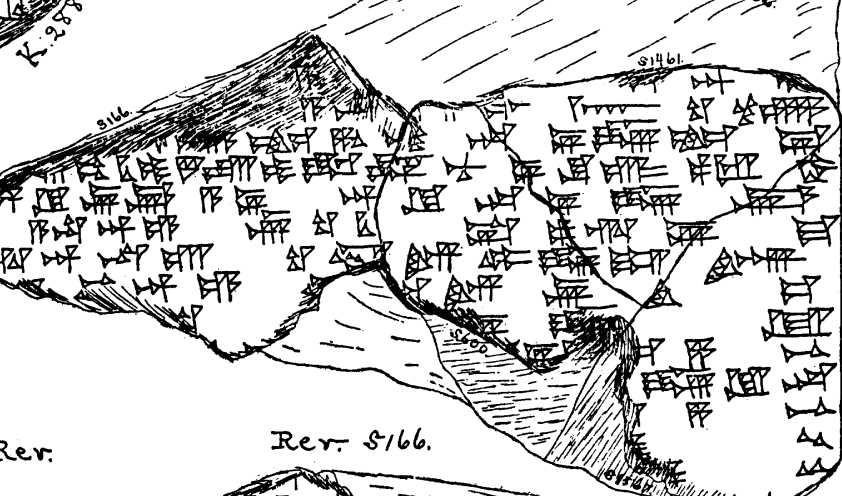

Ke2883 Rer.

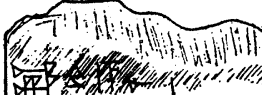

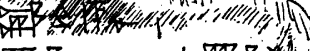

辰

$\Delta$ 然

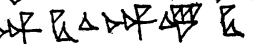

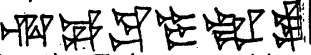

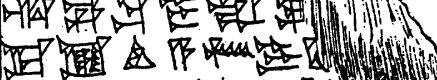

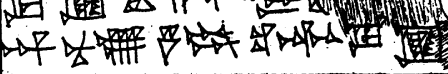

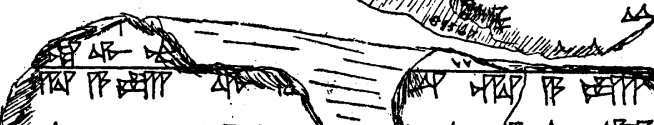

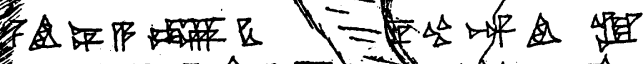

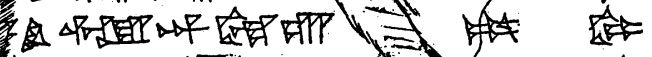

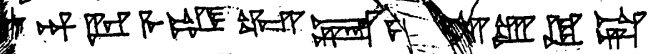

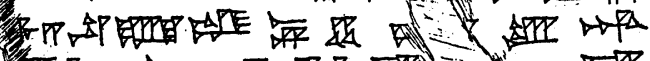

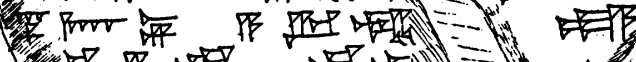
$\leftrightarrow 4 \Rightarrow$ 盾

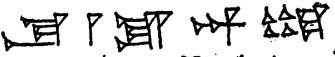

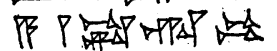

$\mathrm{K} 2132$

K2132, bidge.

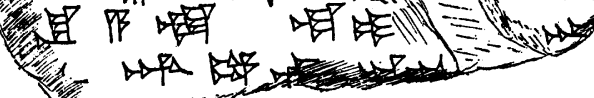

等

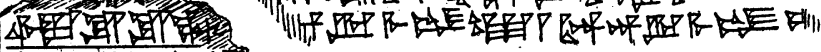

$K_{278}\left(+K_{1944}+K_{9064}\right)$.

G

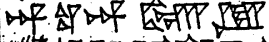
近进

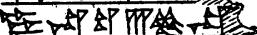

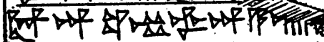

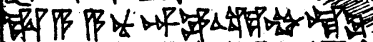

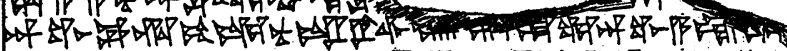

T2

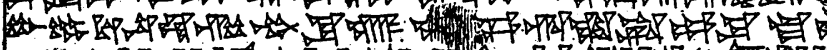

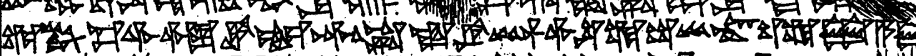

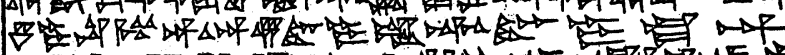

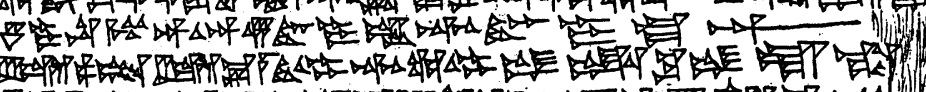

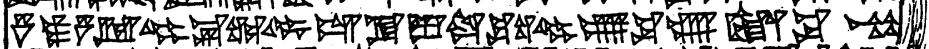

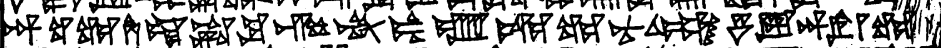

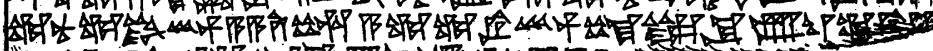

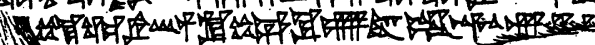

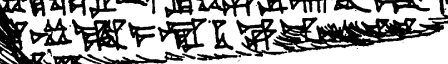


Plate XIII

Born. 129.

Qbu

\begin{tabular}{|c|c|}
\hline 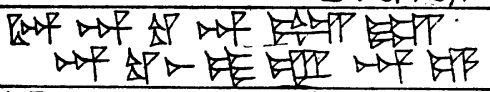 & 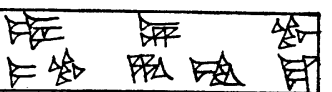 \\
\hline 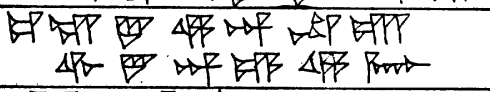 & 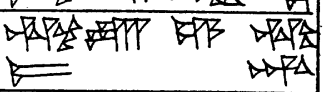 \\
\hline 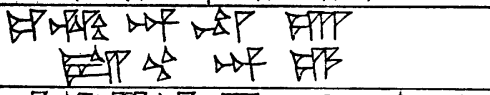 & $F$ \\
\hline
\end{tabular}

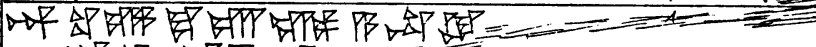

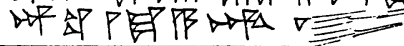

$\Leftrightarrow$ 近

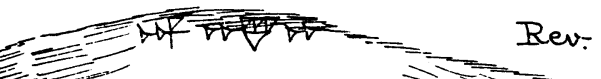

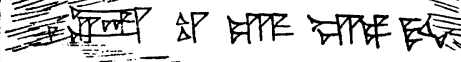

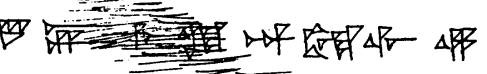

$\Delta \Delta \Delta \Delta \Delta D_{\Delta}$

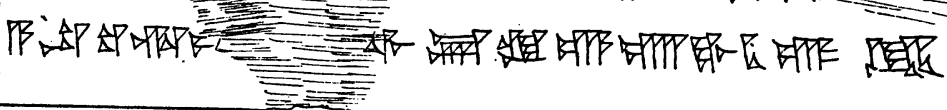

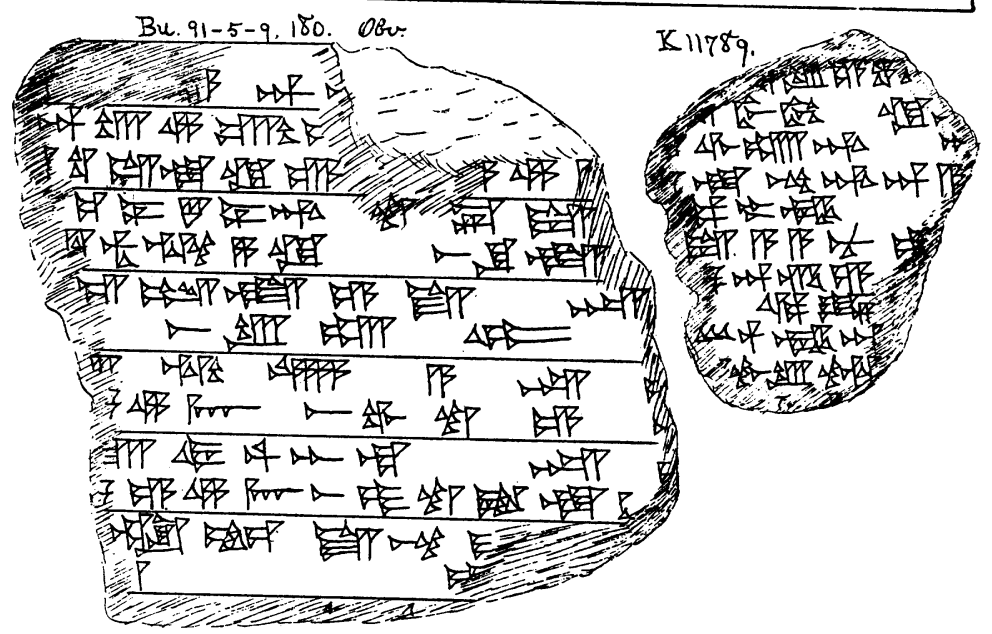

K4795. Obo.

1

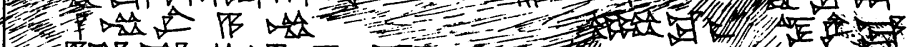

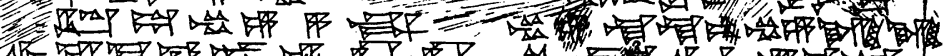

4K

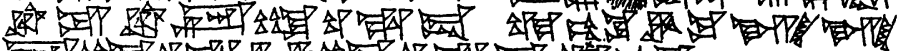

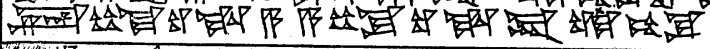

y

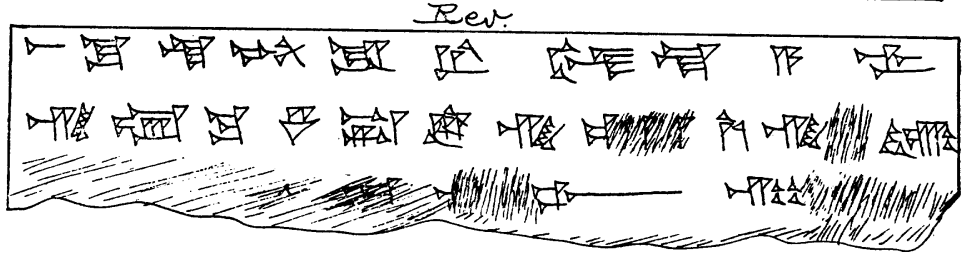

This content downloaded from 129.219.247.033 on September 01, 2016 14:07:24 PM All use subject to University of Chicago Press Terms and Conditions (http://www.journals.uchicago.edu/t-and-c). 
Plate XIV.

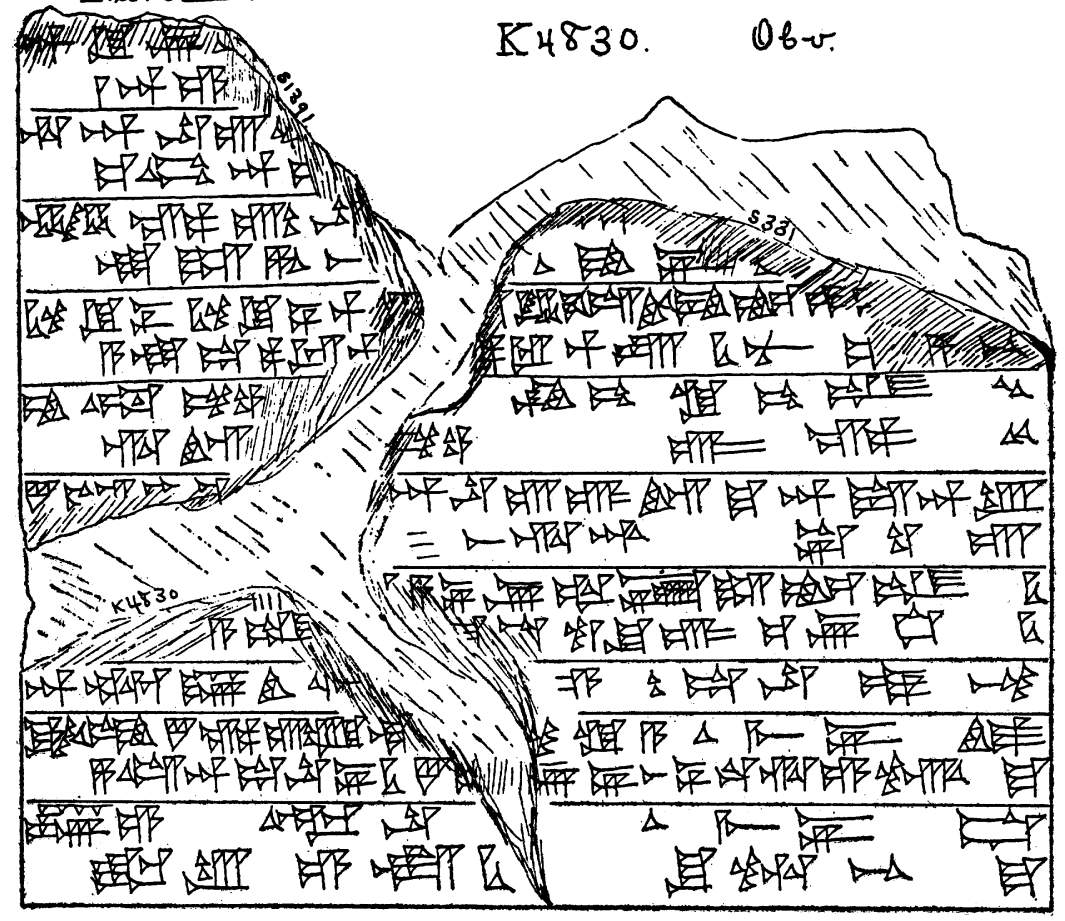

\section{Rer.}

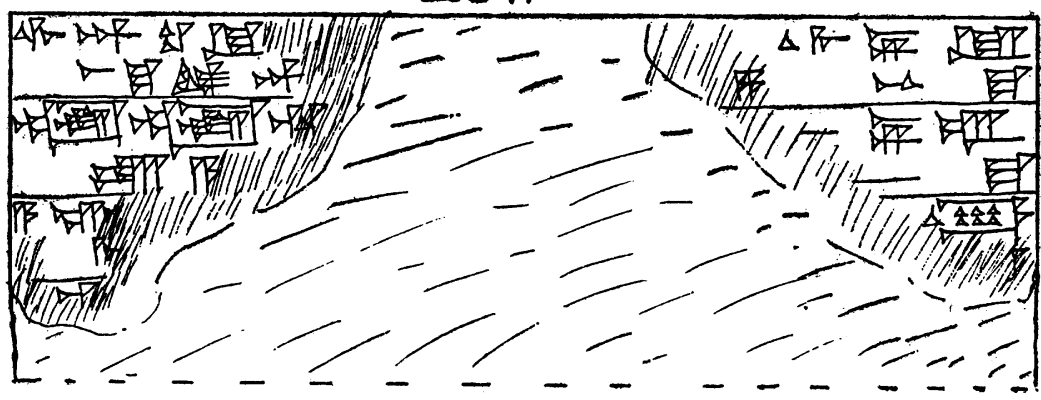

K 4922. Obo.

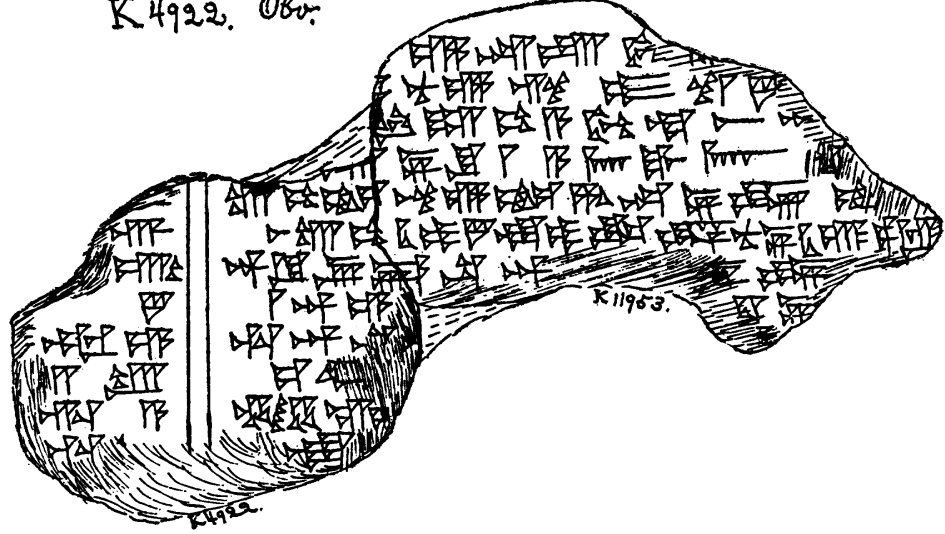




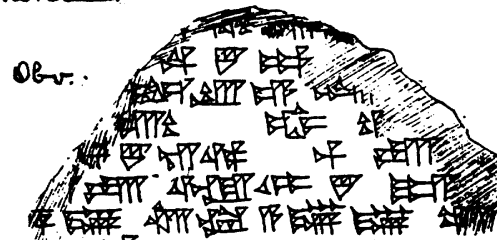
it

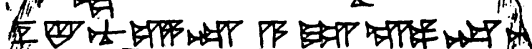

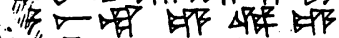

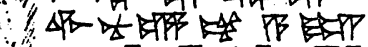

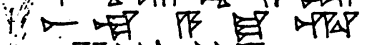

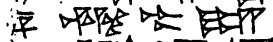

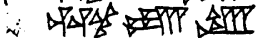

陌

3 的度

$\mathrm{K} 4654$.

TRAB

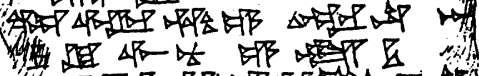

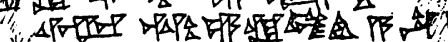

UTR $\triangle P F \square$ D

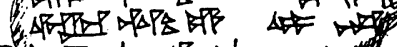

A

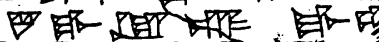

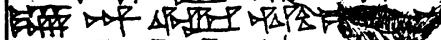

- T2 47 T

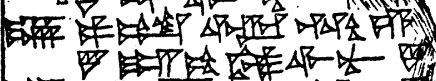

4 叶

Y 14075

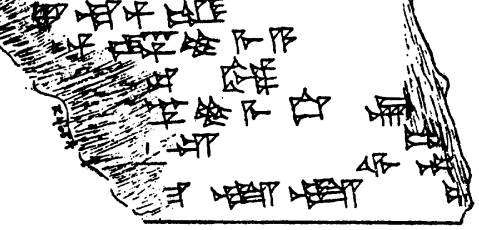

K5069. Obo

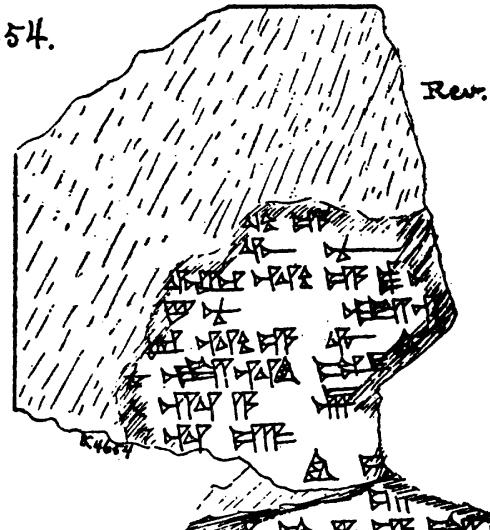

-

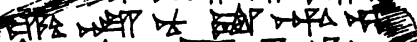

t)

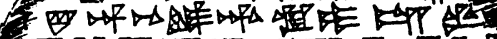

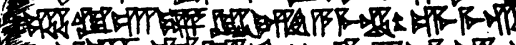

3.

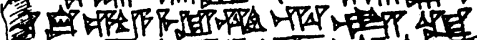

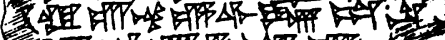

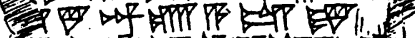

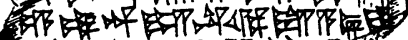

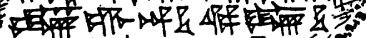

的

售 $P$ 阝

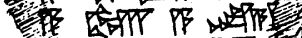

箠

管

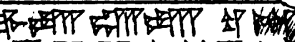

K 3462

Reve

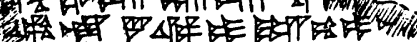

3.

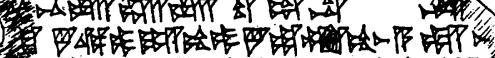
4 4 i

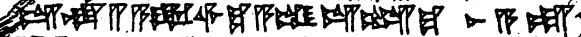

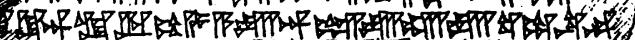

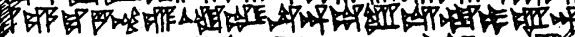
3 -

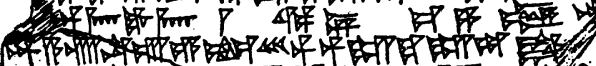

$\rightarrow$ tras

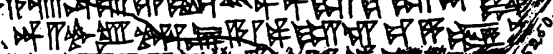

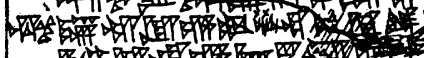

FA

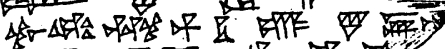

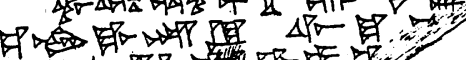

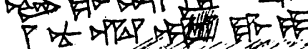

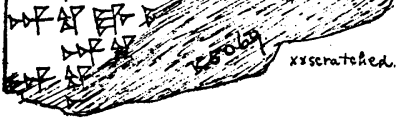

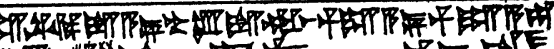
保性 (10) 4.

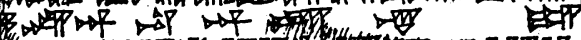

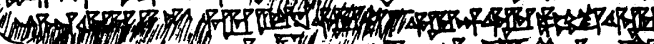
(A)

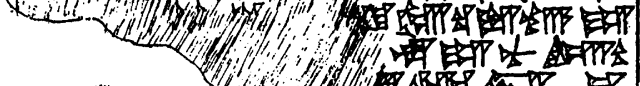

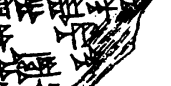

K.3462 ofv:
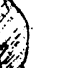

$x x$

innothad.

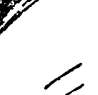
$1>1$

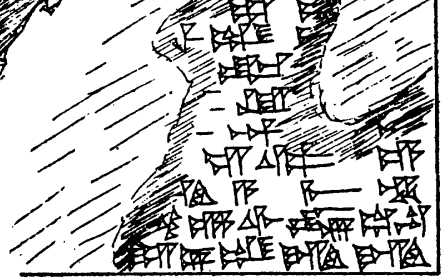


Plate XVI.

K 3138.

Obre

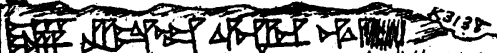

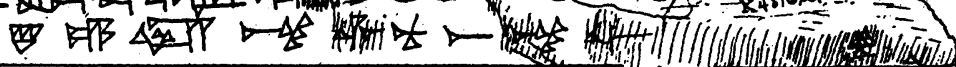

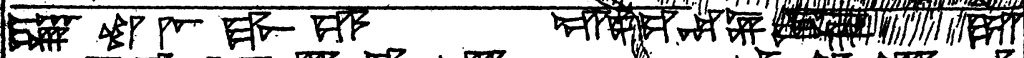

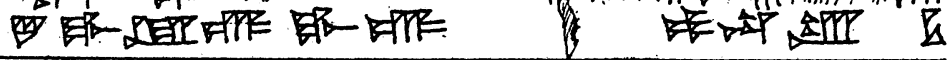

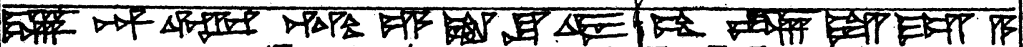

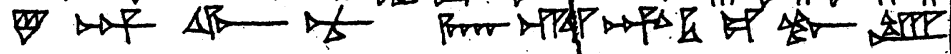
A

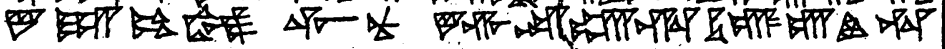

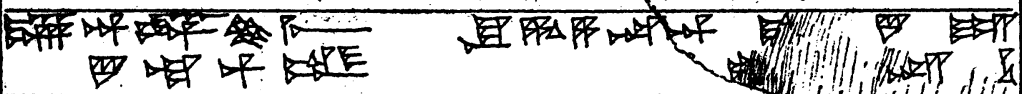

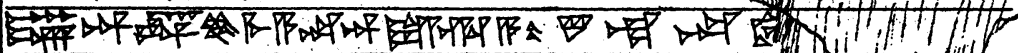
1007 (2) MPP PAP

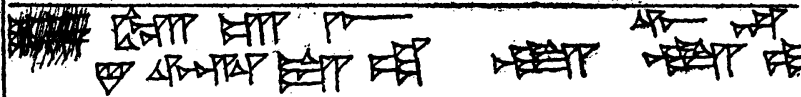

\section{Rev.}

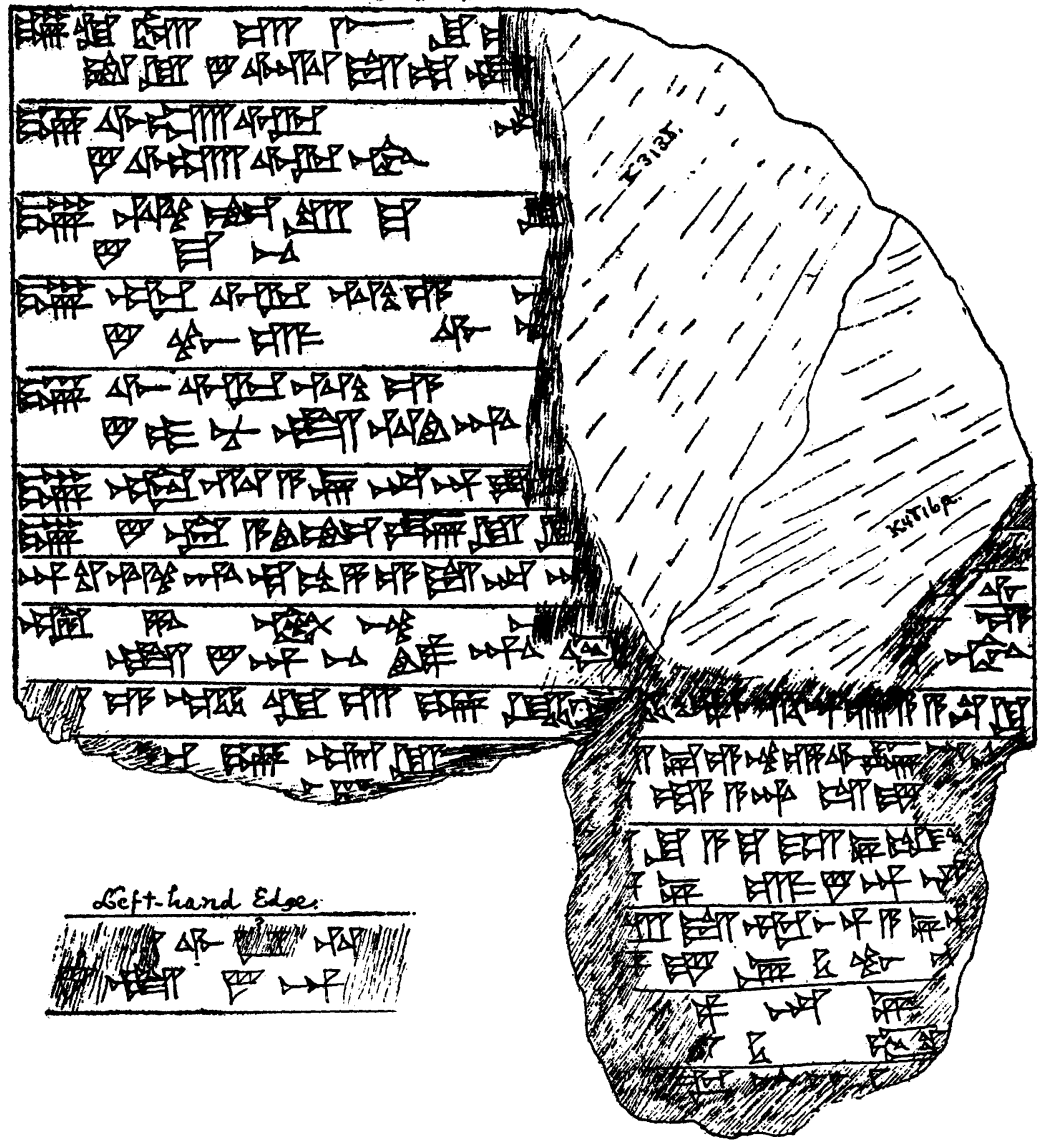



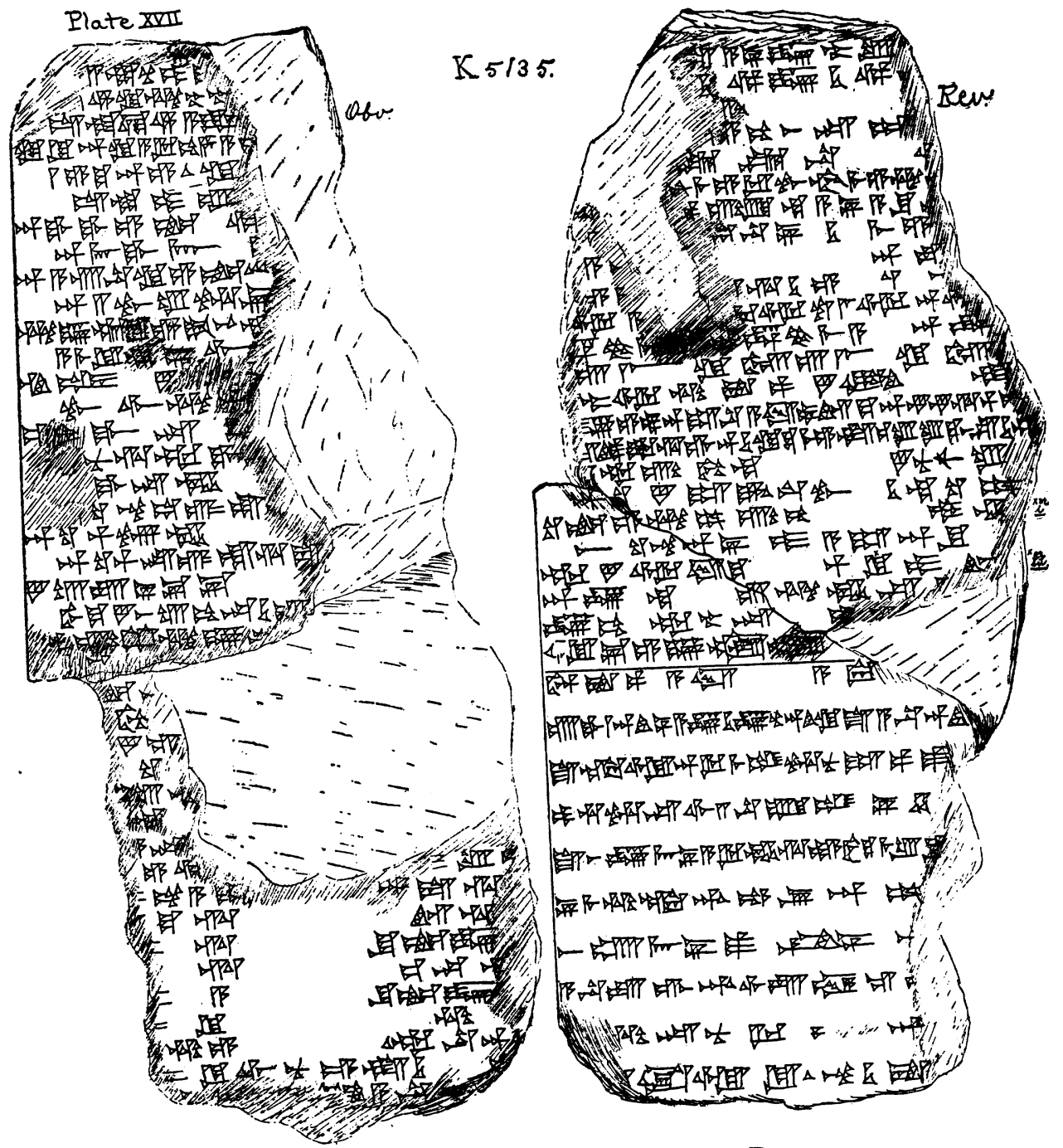

$\overline{\mathrm{X}} 4986$.

Dbu.

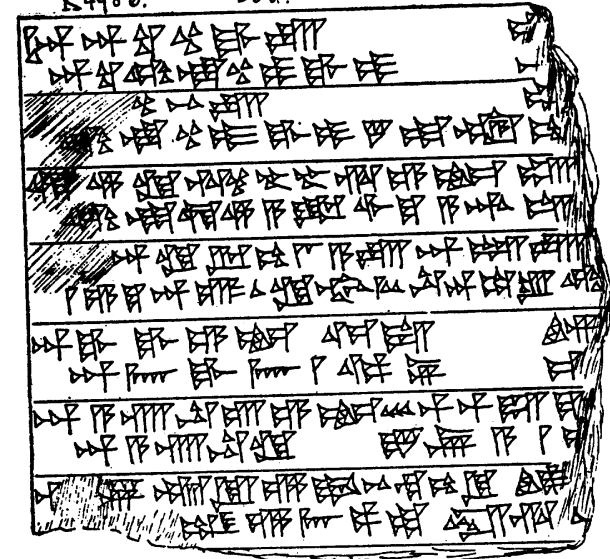

Rev.

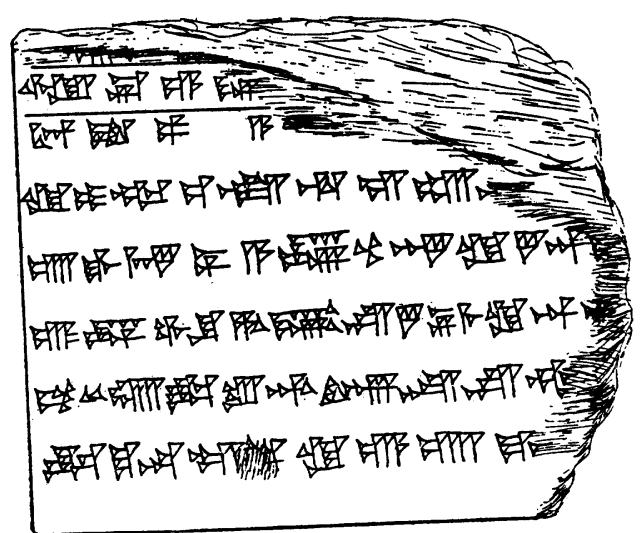


Plate XVIII

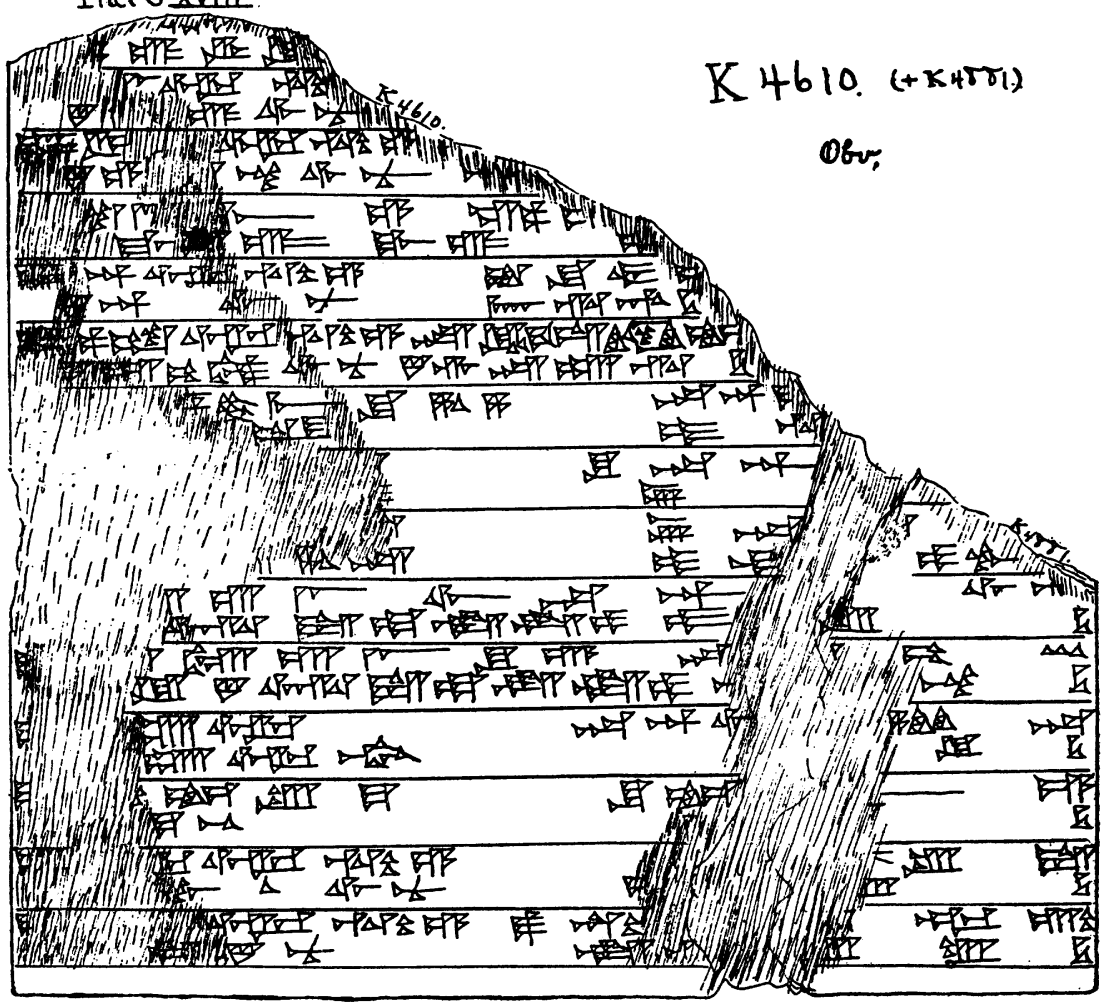

Reve

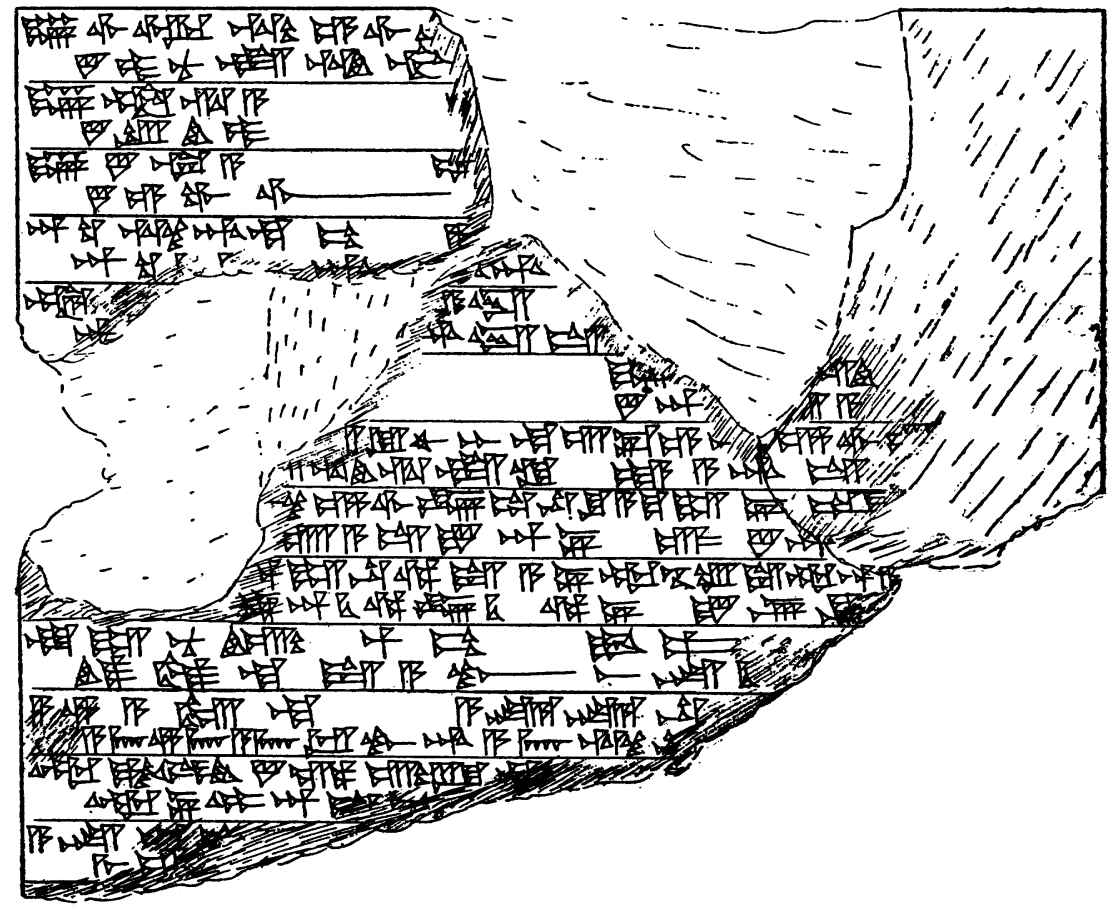

[HEBraICA, XVII]

This content downloaded from 129.219.247.033 on September 01, 2016 14:07:24 PM All use subject to University of Chicago Press Terms and Conditions (http://www.journals.uchicago.edu/t-and-c). 
Plate XIX.

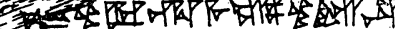

310 E

S1033. Obo

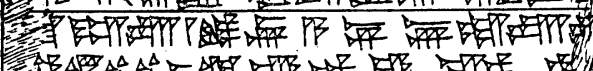

3

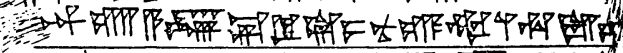

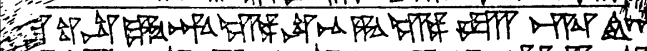

T)

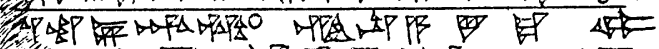

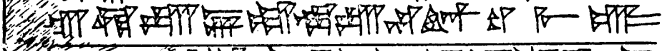

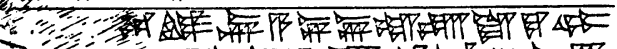

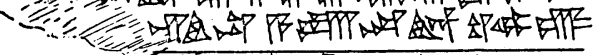

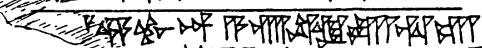

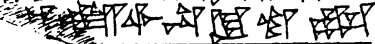

1及W7

$83-1-18,472$. Qbr.

Rev.

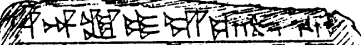

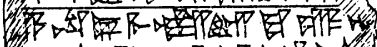

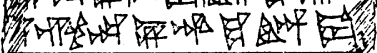

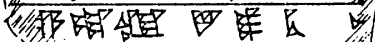

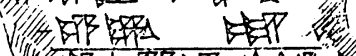

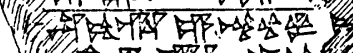

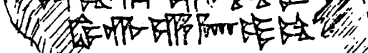
(

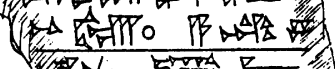

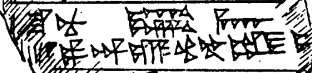

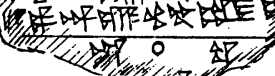

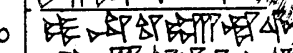

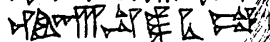
APp E

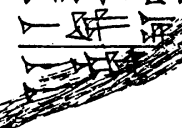
(n)

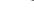
$-1$ $-$ 到 .

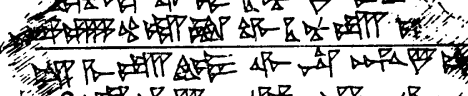
O

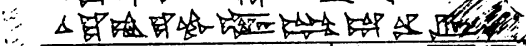
等 氛 WF A

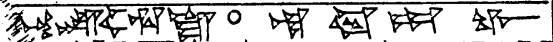

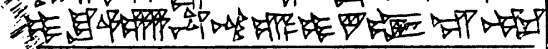

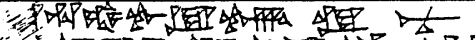

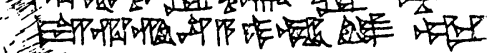

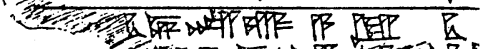
织

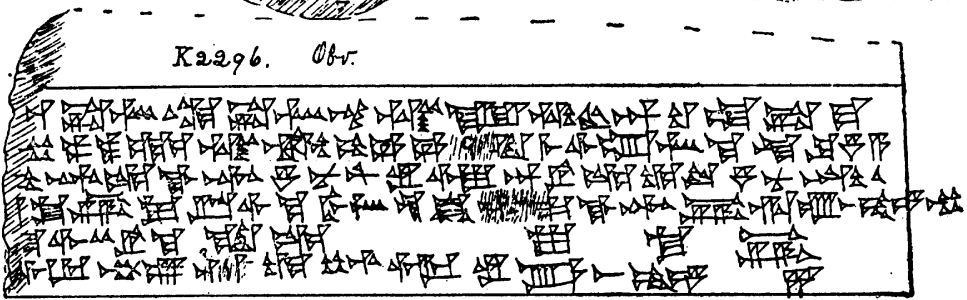

Rev.

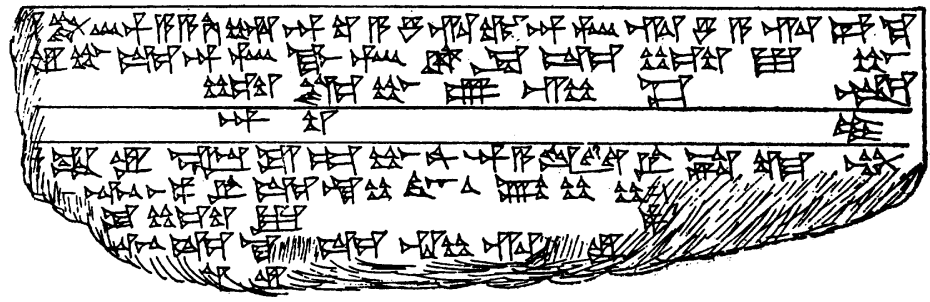


FP 05248 . Rev

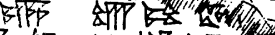

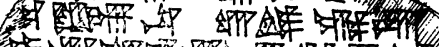

6 ATP

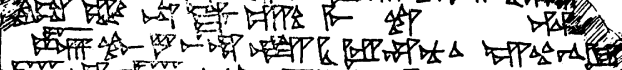

蓶

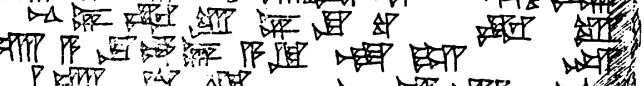

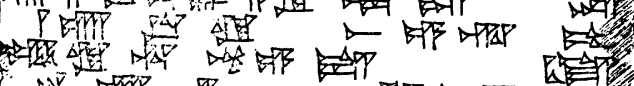

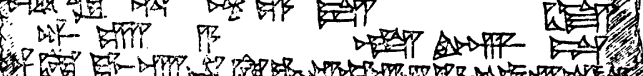

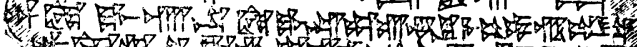

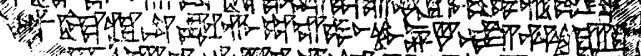

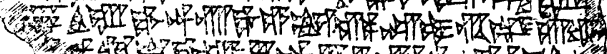

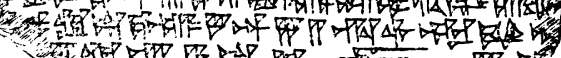

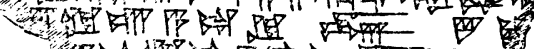

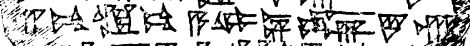

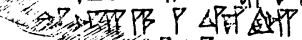

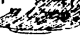

Bu. $91-\div-9,132$. OQve

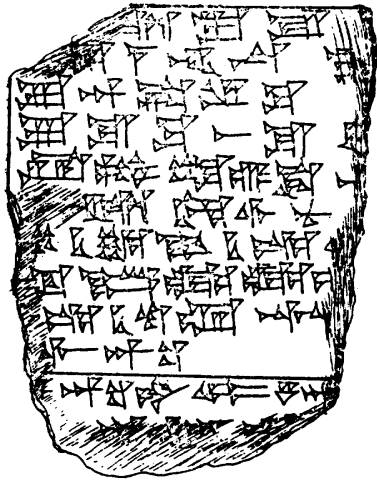

Rev.

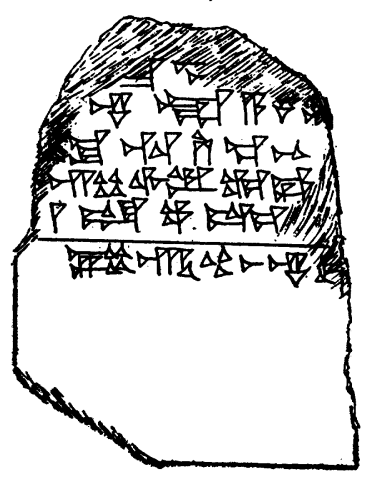

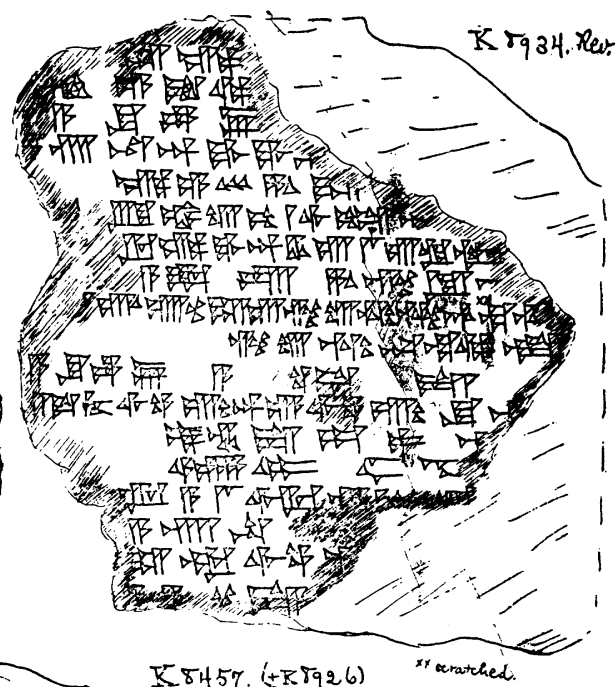

Qbe. 